\title{
Stellar energetic particle ionization in protoplanetary disks around T Tauri stars
}

\author{
Ch. Rab ${ }^{1}$, M. Güdel ${ }^{1}$, M. Padovani ${ }^{2}$, I. Kamp ${ }^{3}$, W.-F. Thi ${ }^{4}$, P. Woitke ${ }^{5}$, and G. Aresu ${ }^{6}$ \\ 1 University of Vienna, Dept. of Astrophysics, Türkenschanzstr. 17, 1180 Wien, Austria \\ e-mail: christian.rab@univie.ac.at \\ 2 INAF-Ossevatorio Astrofisico di Arcetri, Largo E. Fermi, 5, 50125 Firenze, Italy \\ 3 Kapteyn Astronomical Institute, University of Groningen, PO Box 800, 9700 AV Groningen, The Netherlands \\ ${ }_{5}^{4}$ Max-Planck-Institut für extraterrestrische Physik, Giessenbachstrasse 1, 85748 Garching, Germany \\ 5 SUPA, School of Physics \& Astronomy, University of St. Andrews, North Haugh, St. Andrews KY16 9SS, UK \\ 6 INAF, Osservatorio Astronomico di Cagliari, via della Scienza 5, 09047 Selargius, Italy
}

Received 13 December 2016 / Accepted 23 February 2017

\begin{abstract}
Context. Anomalies in the abundance measurements of short lived radionuclides in meteorites indicate that the protosolar nebulae was irradiated by a large number of energetic particles $(E \gtrsim 10 \mathrm{MeV})$. The particle flux of the contemporary Sun cannot explain these anomalies. However, similar to T Tauri stars the young Sun was more active and probably produced enough high energy particles to explain those anomalies.

Aims. We aim to study the interaction of stellar energetic particles with the gas component of the disk (i.e. ionization of molecular hydrogen) and identify possible observational tracers of this interaction.

Methods. We used a 2D radiation thermo-chemical protoplanetary disk code to model a disk representative for $\mathrm{T}$ Tauri stars. We used a particle energy distribution derived from solar flare observations and an enhanced stellar particle flux proposed for T Tauri stars. For this particle spectrum we calculated the stellar particle ionization rate throughout the disk with an accurate particle transport model. We studied the impact of stellar particles for models with varying X-ray and cosmic-ray ionization rates.

Results. We find that stellar particle ionization has a significant impact on the abundances of the common disk ionization tracers $\mathrm{HCO}^{+}$and $\mathrm{N}_{2} \mathrm{H}^{+}$, especially in models with low cosmic-ray ionization rates (e.g. $10^{-19} \mathrm{~s}^{-1}$ for molecular hydrogen). In contrast to cosmic rays and X-rays, stellar particles cannot reach the midplane of the disk. Therefore molecular ions residing in the disk surface layers are more affected by stellar particle ionization than molecular ions tracing the cold layers and midplane of the disk.

Conclusions. Spatially resolved observations of molecular ions tracing different vertical layers of the disk allow to disentangle the contribution of stellar particle ionization from other competing ionization sources. Modelling such observations with a model like the one presented here allows to constrain the stellar particle flux in disks around T Tauri stars.
\end{abstract}

Key words. stars: formation - circumstellar matter - stars: activity - radiative transfer - astrochemistry - methods: numerical

\section{Introduction}

Our Sun acts as a particle accelerator and produces energetic particles with energies $\geq 10 \mathrm{MeV}$ (e.g. Mewaldt et al. 2007). Such particles are also called solar cosmic rays as their energies are comparable to Galactic cosmic rays. They are accelerated in highly violent events like flares and/or close to the solar surface due to shocks produced by coronal mass ejections (Reames 2015). Therefore the energetic particle flux is strongly correlated with the activity of the Sun (e.g. Mewaldt et al. 2005; Reedy 2012).

From X-ray observations of T Tauri stars we know that their X-ray luminosities can be up to $10^{4}$ times higher than the $\mathrm{X}$-ray luminosity of the contemporary Sun (e.g. Feigelson \& Montmerle 1999; Güdel et al. 2007). Such high X-ray luminosities are rather a result of enhanced flare activity of young stars than coronal effects (e.g Feigelson et al. 2002). Enhanced activity of T Tauri stars implies an increase of their stellar energetic particle (SP) flux. From simple scaling with the X-ray luminosity and considering that young stars produce more powerful flares, Feigelson et al. (2002) derived a typical SP flux for T Tauri stars $\approx 10^{5}$ times higher than for the contemporary Sun. Under these assumptions T Tauri stars might show on average a continuous proton flux of $f_{\mathrm{p}}\left(E_{\mathrm{p}} \geq 10 \mathrm{MeV}\right) \approx 10^{7}$ protons $\mathrm{cm}^{-2} \mathrm{~s}^{-1}$ at a distance of 1 au from the star.

Such a scenario is also likely for the young Sun. Measurements of decay products of short-lived radionuclides (SLR) like ${ }^{10} \mathrm{Be}$ or ${ }^{26} \mathrm{Al}$ in meteorites indicate an overabundance of SLRs in the early phases of our solar system (e.g. Meyer \& Clayton 2000). One likely explanation for these abundance anomalies are spallation reactions of SPs with the dust in the protosolar nebula (e.g. Lee et al. 1998b; McKeegan et al. 2000; Gounelle et al. 2001, 2006). However, this would require a strongly enhanced SP flux of the young Sun by a factor $\gtrsim 3 \times 10^{5}$ compared to the contemporary Sun (McKeegan et al. 2000), consistent with the estimated SP flux of T Tauri stars derived by Feigelson et al. (2002).

More recently Ceccarelli et al. (2014) reported a first indirect measurement of SPs in the protostar OMC-2 FIR 4. They observed a low $\mathrm{HCO}^{+} / \mathrm{N}_{2} \mathrm{H}^{+}$abundance ratio between three and four that requires high $\mathrm{H}_{2}$ ionization rates $>10^{-14} \mathrm{~s}^{-1}$ throughout the protostellar envelope. They explain this high ionization rate by the presence of SPs. From this ionization rate they derived a particle flux of $f_{\mathrm{p}}\left(E_{\mathrm{p}} \geq 10 \mathrm{MeV}\right) \geq 3-9 \times 10^{11}$ protons cm $\mathrm{cm}^{-2} \mathrm{~s}^{-1}$ 
at 1 au distance from the star. Such a high flux would be more than sufficient to explain the over-abundance of SLRs in the solar nebula.

A further indication of SPs in young stars is the anticorrelation of X-ray fluxes with the crystalline mass fraction of the circumstellar dust found by Glauser et al. (2009). They argue that SPs are responsible for the amorphization of dust particles and that the correlation can be explained if the SP flux scales with the stellar X-ray luminosity. Trappitsch \& Ciesla (2015) tested such a scenario by using detailed models of SP transport for a protoplanetary disk (i.e. SP flux as a function of height of the disk). They also considered the vertical "mixing" of dust particles. According to their models SP irradiation of the disk cannot explain the total SLR abundances in the solar nebula but might play a role for dust amorphization.

Like Galactic cosmic rays SPs not only interact with the solid component but also with the gas component of the disk. However, little is known about the impact of SPs on the chemical structure of disks. Turner \& Drake (2009) investigated the relevance of SPs on the size of dead-zones in disks. Assuming similar enhancement factors as mentioned above they find that SPs can decrease the size of the dead zone depending on the disk model and other ionization sources.

We present a first approach to study the impact of SP ionization on the chemical structure of the disk. We assume a typical SP flux as proposed for T Tauri stars to study the impact of SP ionization on the common disk ionization tracers $\mathrm{HCO}^{+}$ and $\mathrm{N}_{2} \mathrm{H}^{+}$. We use the radiation thermo-chemical disk model PRODiMo (PROtoplanetary DIsk MOdel, Woitke et al. 2009, 2016; Kamp et al. 2010; Thi et al. 2011) to model the thermal and chemical structure of the disk. We argue that spatially resolved radial intensity profiles of molecular ion emission of the disk allow to constrain the SP flux of T Tauri stars.

In Sect. 2 we describe our method to derive the ionization rate due to SPs and the disk model. Our results are presented in Sect. 3 where we show the impact of SPs on the common disk ionization tracers $\mathrm{HCO}^{+}$and $\mathrm{N}_{2} \mathrm{H}^{+}$. In Sect. 4 we discuss possibilities to constrain the SP flux via observations of molecular ion emission and future prospects for modelling of SP ionization in protoplanetary disks. We present a summary and our main conclusions in Sect. 5.

\section{Method}

To investigate the impact of SPs on the disk chemical structure we first needed to determine the SP flux and the particle energy distribution. With these particle spectra we were able to calculate the ionization rate throughout the disk. We applied this to a disk structure representative for disks around T Tauri stars. With the radiation thermo-chemical disk code PRODIMo we calculated the chemical abundances. We did this for a series of models in which we also considered other important high energy ionization sources like Galactic cosmic rays (CR) and X-rays.

\subsection{Stellar energetic particle spectra}

As the actual particle spectra and fluxes of young stars are unknown, we derived the spectra from the knowledge available from our Sun. The origin of solar energetic particles are most likely flares and/or shock waves driven by coronal-mass ejections (CME; Reames 2013, 2015). Flares act like point sources on the solar surface whereas the shock waves can fill half of the heliosphere at around 2 Solar radii (Reames 2015). Particle

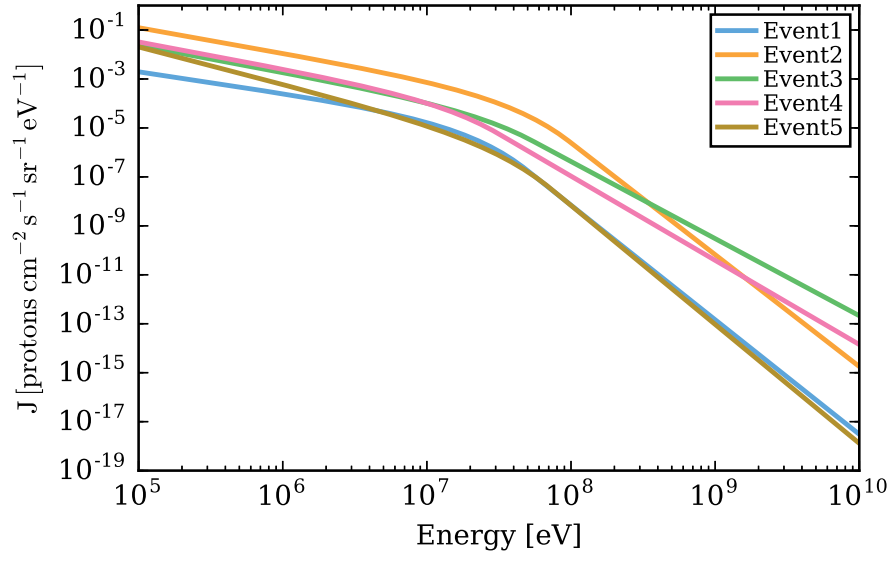

Fig. 1. Stellar particle (proton) spectra for five different solar particle events. Shown are fits to the measurements presented in Mewaldt et al. (2005).

fluxes are not continuous but rather produced in events lasting from several hours to days (Feigelson et al. 2002; Mewaldt et al. 2005).

Based on observed X-ray luminosities of solar analogs in the Orion nebula Feigelson et al. (2002) estimated that SP fluxes in young stars are likely $\approx 10^{5}$ times higher than in the contemporary Sun (see also Glassgold et al. 2005). As T Tauri stars are very active Feigelson et al. (2002) argue that it is likely that $\mathrm{X}$-ray flares with luminosities below the detection limit occur several times a day (the same argument holds for CMEs). In that case the X-ray flares and consequently also SP events overlap, resulting in an enhanced continuous SP flux.

Based on these arguments we have assumed here a continuous and enhanced SP flux for young T Tauri stars. This approximation is consistent with the assumption of powerful and overlapping flare and CME events of T Tauri stars.

In Mewaldt et al. (2005) measurements of five different solar particle events are reported. We used their fitting formulae (see their Eq. (2) and Table 5) and derive SP spectra (protons in this case) averaged over the duration of the observed events. These measurements are for particles with energies up to several $100 \mathrm{MeV}$. We extrapolated their results up to energies typical for Galactic cosmic rays of $\approx 10 \mathrm{GeV}$. This is consistent with the maximum energy $E_{\max } \approx 30 \mathrm{GeV}$ derived by Padovani et al. $(2015,2016)$ for particles accelerated on protostellar surfaces. The resulting spectra are shown in Fig. 1. The flux levels for the different events can vary by up to two orders of magnitude and there is also some variation in the shape of the spectra.

Reedy (2012) reported proton fluxes of the contemporary Sun for five solar cycles. Typical values for the cycle averaged fluxes at a distance of $1 \mathrm{au}$ are $f_{\mathrm{p}}\left(E_{\mathrm{p}}>10 \mathrm{MeV}\right)=$ 59-213 protons $\mathrm{cm}^{-2} \mathrm{~s}^{-1}$. For the Eventl spectrum in Fig. 1 we get $f_{\mathrm{p}}\left(E_{\mathrm{p}}>10 \mathrm{MeV}\right)=151$ protons $\mathrm{cm}^{-2} \mathrm{~s}^{-1}$ at $1 \mathrm{au}$, very similar to the reported values of Reedy (2012).

Here we used only the Eventl spectrum, which we call the "active Sun" spectrum. We simply scaled the active Sun spectrum by a factor of $\approx 10^{5}$, as proposed by Feigelson et al. (2002), to get a typical "active T Tauri" spectrum. The resulting SP flux of $f_{\mathrm{p}}\left(E_{\mathrm{p}}>10 \mathrm{MeV}\right)=1.51 \times 10^{7}$ protons $\mathrm{cm}^{-2} \mathrm{~s}^{-1}$ is consistent with the value of $\approx 10^{7}$ protons $\mathrm{cm}^{-2} \mathrm{~s}^{-1}$ derived by Feigelson et al. (2002) for young solar analogs in the Orion Nebula cluster. The two SP spectra are shown in Fig. 2, where we also show two cases of Galactic cosmic-ray spectra for comparison (see Sect. 2.3.2). 


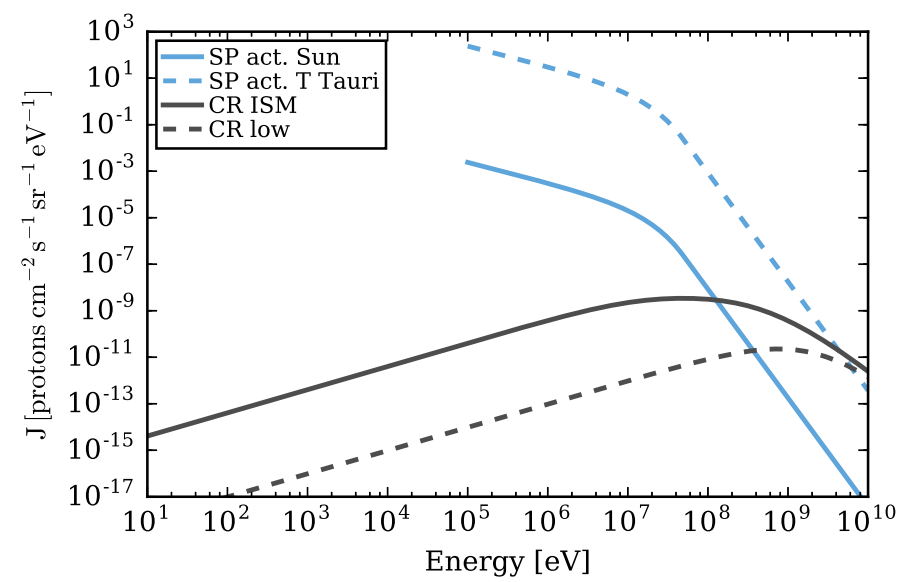

Fig. 2. Stellar energetic proton (SP) and cosmic-ray (CR) input spectra. The blue solid and dashed lines show the active Sun and active T Tauri SP spectrum, respectively. The black solid line shows the "LIS W98" CR spectrum from Webber (1998) and the dashed black line the attenuated "Solar Max" CR spectrum from Cleeves et al. (2013).

For comparison we also present models applying the same approach for the treatment of SPs as Turner \& Drake (2009). For their model they assumed that SPs behave very similar to Galactic CRs (e.g. particle energies). The details of the Turner model are discussed in Appendix D.

It is not clear if SPs actually reach the disk (see Feigelson et al. 2002, for a discussion). However, as we are interested in the possible impact of SPs on the chemistry, we simply assumed that all SPs reach the disk. We further discuss this assumption in Sect. 4.3.

\subsection{Stellar particle transport and ionization rate}

Energetic particles hitting the disk interact with its gas and dust contents. Although the interaction with the solids is relevant for the production of SLR, here we are only interested in the interaction with the gas. Dust only plays a minor role in the actual attenuation of particles as only $\approx 1 \%$ of the mass in protoplanetary disk is in solids (see also Trappitsch \& Ciesla 2015). Very similar to Galactic cosmic rays, SPs mainly ionize the gaseous medium (i.e. molecular hydrogen). Energetic particles interact multiple times and ionize many atoms and molecules on their way until they eventually have lost their energy completely. This complex process requires detailed particle transport models.

To model the transport of energetic particles through the disk gas, we used the continuous slowing down approximation, which assumes that particles lose an infinitesimal fraction of their energy during propagation (Takayanagi 1973). We used the results obtained by Padovani et al. (2009, 2013a) who compute the propagation of CRs in a 1D slab, taking all the relevant energy loss processes into account. They give a useful fitting formula for the ionization rate as a function of the column density of molecular hydrogen.

In order to apply the results of this 1D transport model, we assumed that SPs travel along straight lines (i.e. no scattering due to their high energies) and that they originate from a point source (the star). We also disregarded the effect of magnetic fields that could increase or decrease the ionization rate depending on their configuration (Padovani \& Galli 2011; Padovani et al. 2013b, see also Sect. 4.3.2).

From the detailed 1D particle transport model we derived a simple fitting formulae for the two SP spectra considered here.
Table 1. Fitting parameters for the stellar particle ionization rate for the two different input spectra.

\begin{tabular}{lcccc}
\hline \hline Name & $\begin{array}{c}\zeta_{\mathrm{L}} \\
\left(\mathrm{s}^{-1}\right)\end{array}$ & $a$ & $\begin{array}{c}\zeta_{\mathrm{H}} \\
\left(\mathrm{s}^{-1}\right)\end{array}$ & $b$ \\
\hline SP active Sun & $1.06(-12)^{a}$ & -0.61 & $8.34(-7)$ & -2.61 \\
SP active T Tauri & $1.06(-7)$ & -0.61 & $8.34(-2)$ & -2.61 \\
\hline
\end{tabular}

Notes. The values provided are for an unattenuated SP ionization rate at 1 au distance from the star (Eqs. (1) and (2)). ${ }^{(a)} x(y)$ means $x \times 10^{y}$.

The SP ionization rate $\zeta_{\mathrm{SP}}$ for molecular hydrogen as a function of the total hydrogen column density $N_{\langle\mathrm{H}\rangle}=N_{\mathrm{H}}+2 \times N_{\mathrm{H}_{2}}$ is given by

$\zeta_{\mathrm{SP}}\left(N_{\langle\mathrm{H}\rangle}\right)=\left[\frac{1}{\zeta_{\mathrm{L}}\left(\frac{N_{\mathrm{H}\rangle}}{10^{20} \mathrm{~cm}^{-2}}\right)^{a}}+\frac{1}{\zeta_{\mathrm{H}}\left(\frac{N_{\langle\mathrm{H}\rangle}}{10^{20} \mathrm{~cm}^{-2}}\right)^{b}}\right]^{-1}\left[\mathrm{~s}^{-1}\right]$,

and for $N_{\langle\mathrm{H}\rangle}>N_{\mathrm{E}}$ by

$\zeta_{\mathrm{SP}, \mathrm{E}}\left(N_{\langle\mathrm{H}\rangle}\right)=\zeta_{\mathrm{SP}}\left(N_{\langle\mathrm{H}\rangle}\right) \times \exp \left[-\left(\frac{N_{\langle\mathrm{H}\rangle}}{N_{\mathrm{E}}}-1.0\right)\right]\left[\mathrm{s}^{-1}\right]$.

The two power laws in Eq. (1) are a consequence of the shape of the SP input spectra (see Fig. 2). The two parts of Eq. (1) account for the ionization rate at low $\left(\zeta_{\mathrm{L}}\right)$ and high $\left(\zeta_{\mathrm{H}}\right)$ column densities. Equation (2) accounts for the exponential drop of the SP ionization rate starting at a certain column density given by $N_{\mathrm{E}}$ (i.e. similar to CRs). For the two SP spectra considered here $N_{\mathrm{E}}=2.5 \times 10^{25} \mathrm{~cm}^{-2}$. The other fitting parameters $\zeta_{\mathrm{L}}, \zeta_{\mathrm{H}}, a$ and $b$ are given in Table 1.

Equations (1) and (2) provide the unattenuated SP ionization rate at 1 au distance from the star (i.e. for a SP flux at $1 \mathrm{au}$ ). To account for geometric dilution we scaled $\zeta_{\mathrm{SP}}\left(N_{\langle\mathrm{H}\rangle}\right)$ by $1 / r^{2}$ at every point in the disk ( $r$ is the distance to the star in au). For the chemistry we simply added $\zeta_{\mathrm{SP}}$ to the ionization rate for Galactic cosmic rays $\zeta_{\mathrm{CR}}$ (see Sect. 2.3.2).

\subsection{Other ionization sources}

To investigate the impact of SPs on the disk ionization structure also other ionization sources common to T Tauri stars must be considered. Besides SPs our model includes stellar UV and $\mathrm{X}$-ray radiation, interstellar UV radiation and Galactic cosmic rays (CRs). However, most relevant for our study are the high energy ionization sources capable of ionizing molecular hydrogen: SPs, X-rays and CRs.

\subsubsection{X-rays}

To model the stellar X-ray spectrum we used an approximation for an isothermal bremsstrahlung spectrum (Glassgold et al. 1997; Aresu et al. 2011)

$F(E) \propto \frac{1}{E} \exp \left(-E / k T_{\mathrm{X}}\right)$

where $E$ is the photon energy (here in the range of 0.1 to $20 \mathrm{keV}$ ), $k$ the Boltzmann constant and $T_{\mathrm{X}}$ is the plasma temperature. This spectrum is scaled to a given total X-ray luminosity $L_{\mathrm{X}}(0.3 \leq$ $E \leq 10 \mathrm{keV}$, e.g. Güdel et al. 2010).

As a result of the activity of the stars (e.g. flares) X-ray radiation of young stars is variable. We account for this in a simple way by including a spectrum with a X-ray luminosity and 


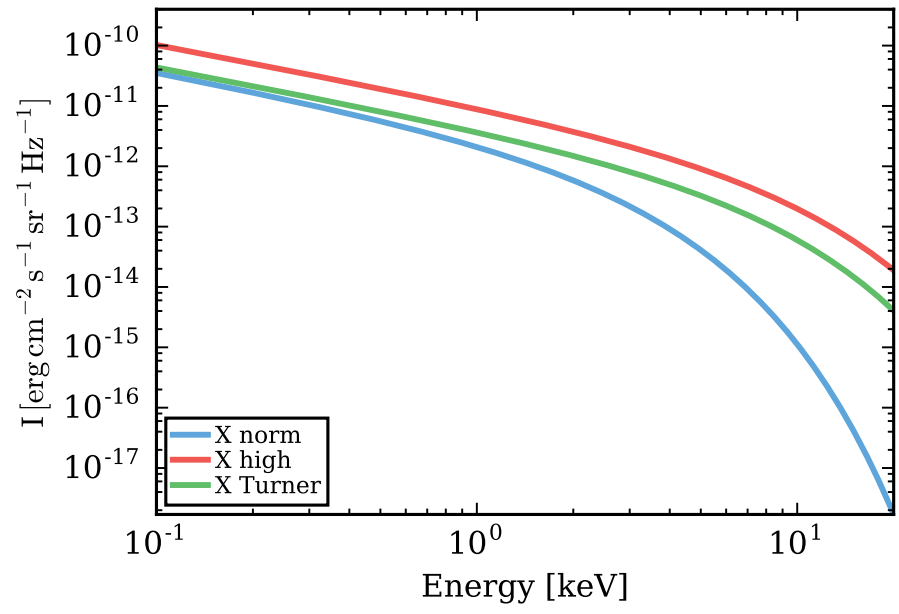

Fig. 3. Stellar X-ray input spectra (see Table 2).

Table 2. Parameters for the stellar X-ray input spectra (see Fig. 3).

\begin{tabular}{lccc}
\hline \hline Name & Spectrum & $\begin{array}{c}L_{\mathrm{X}} \\
\left(\mathrm{erg} \mathrm{s}^{-1}\right)\end{array}$ & $\begin{array}{c}T_{\mathrm{X}} \\
(\mathrm{K})\end{array}$ \\
\hline X norm & typical T Tauri & $1(30)$ & $2(7)$ \\
X high & flared T Tauri & $5(30)$ & $7(7)$ \\
X Turner & Turner \& Drake (2009) & $2(30)$ & $5.8(7)$ \\
\hline
\end{tabular}

temperature representative for a typical T Tauri star, and a spectrum which represents a flaring spectrum (more activity) with higher luminosity and a harder (hotter) radiation. However, we actually ignored the variability and assume time averaged X-ray fluxes. According to Ilgner \& Nelson (2006) this is a reasonable assumption as typically the recombination timescales in the disk are longer than the flaring period and large parts of the disk ( $r \gtrsim 2$ au in their model) respond to an enhanced average X-ray luminosity.

We also included the stellar X-ray properties used in Turner \& Drake (2009) for the Turner models (Appendix D). The parameters for the various X-ray input spectra are given in Table 2 and the spectra are shown in Fig. 3.

To derive the X-ray ionization rate $\zeta_{\mathrm{X}}$ we used X-ray radiative transfer including scattering and a detailed treatment of X-ray chemistry (Aresu et al. 2011; Meijerink et al. 2012). For more details on the new X-ray radiative transfer module in ProDiMo see Appendix A.

\subsubsection{Galactic cosmic rays}

Protoplanetary disks are exposed to Galactic cosmic rays (CR). Differently to SPs and X-rays, CRs are not of stellar origin and hit the disk isotropically. Cleeves et al. (2013) proposed that for $\mathrm{T}$ Tauri disks the actual CR ionization rate might be much lower compared to the interstellar medium (ISM) due to modulation of the impinging CRs by the heliosphere ("T-Tauriosphere").

From modelling molecular ion observations of the TW Hya disk, Cleeves et al. (2015) derived an upper limit for the total $\mathrm{H}_{2}$ ionization rate of $\zeta \lesssim 10^{-19} \mathrm{~s}^{-1}$. This upper limit applies for all ionization sources including SLRs (e.g. Umebayashi \& Nakano 2009). SLR ionization is a potentially important ionization source in the midplane of disks. However, similar to Cleeves et al. (2015) we modelled the low ionization rate scenario by reducing the CR ionization rate and did not explicitly treat SLR ionization in the models presented here.
Table 3. Fitting parameters for the cosmic-ray ionization rate.

\begin{tabular}{lcccc}
\hline \hline Name & $\begin{array}{c}\zeta_{1} \\
\left(\mathrm{~s}^{-1}\right)\end{array}$ & $\begin{array}{c}\zeta_{\mathrm{h}} \\
\left(\mathrm{s}^{-1}\right)\end{array}$ & $\begin{array}{c}\Sigma_{0} \\
\left(\mathrm{~g} \mathrm{~cm}^{-2}\right)\end{array}$ & $a$ \\
\hline ISM CR $^{a}$ & $2(-17)$ & $2.6(-18)$ & 244 & 0.021 \\
low CR & $2(-19)$ & $8.0(-19)$ & 230 & -0.01 \\
\hline
\end{tabular}

Notes. ${ }^{(a)}$ ISM W98 spectrum (Padovani et al. 2009, 2013a); ${ }^{(b)}$ modulated "Solar Max" spectrum (Cleeves et al. 2013)

We considered two different CR input spectra, the canonical local ISM CR spectrum (Webber 1998) and a modulated CR spectrum which accounts for the exclusion of CRs by the "T-Tauriosphere" (Cleeves et al. 2013, 2015). For simplicity we call these two spectra "ISM CR" and "low CR", respectively. To calculate the CR ionization rate $\zeta_{\mathrm{CR}}$ in the disk we applied the fitting formulae provided by Padovani et al. (2013a) and Cleeves et al. (2013):

$\zeta_{\mathrm{CR}}\left(N_{\langle\mathrm{H}\rangle}\right)=\frac{\zeta_{\mathrm{l}} \zeta_{\mathrm{h}}}{\zeta_{\mathrm{h}}\left[N_{\langle\mathrm{H}\rangle} / 10^{20} \mathrm{~cm}^{-2}\right]^{a}+\zeta_{1}\left[\exp \left(\Sigma / \Sigma_{0}\right)-1\right]}$.

For simplicity we have assumed that CRs enter the disk perpendicular to the disk surface. Therefore we used the disk vertical hydrogen column density $N_{\langle\mathrm{H}\rangle \text {,ver }}$ and surface density $\Sigma_{\mathrm{ver}}$ for Eq. (4) to calculate $\zeta_{\mathrm{CR}}$ at every point in the disk. The fitting parameters $\zeta_{1}, \zeta_{\mathrm{h}}, \Sigma_{0}$ and $a$ for the two $\mathrm{CR}$ input spectra are given in Table 3 (see Padovani et al. 2009, 2013a, for details). The typical resulting $\mathrm{H}_{2}$ ionization rates in the disk are $\zeta_{\mathrm{CR}} \approx 2 \times 10^{-17} \mathrm{~s}^{-1}$ for the ISM CR spectrum and $\zeta_{\mathrm{CR}} \approx 2 \times 10^{-19} \mathrm{~s}^{-1}$ for the low CR spectrum (see Sects. 3.1 and 3.2).

\subsection{Disk model}

To model the disk we used the radiation thermo-chemical disk code ProDiMo (Woitke et al. 2009, 2016; Kamp et al. 2010; Thi et al. 2011). ProDiMo solves the wavelength dependent continuum radiative transfer which provides the disk dust temperature and the local radiation field. The gas temperature (the balance between heating and cooling) was determined consistently with the chemical abundances. The chemical network included 235 different species and 3143 chemical reactions (see Appendix B for more details).

We used a disk model representing the main properties of a disk around a typical $\mathrm{T}$ Tauri star. The stellar properties and the disk structure of this model are identical to the so-called reference model presented in Woitke et al. (2016). Here we only provide a brief overview of the disk model and refer the reader to Woitke et al. (2016) for details.

In Fig. 4 we show the gas number density, dust density, the local far-UV (FUV) radiation field, gas temperature, dust temperature and the vertical hydrogen column density for the reference model (model CI_XN, see Sect. 2.5 and Table 5). All relevant disk model parameters are given in Table 4.

Based on the similarity solution for viscous accretion disks, we used an axisymmetric flared gas density structure with a Gaussian vertical profile and a powerlaw with a tapered outer edge for the radial column density profile (e.g. Lynden-Bell \& Pringle 1974; Andrews et al. 2009). The vertical scale height as a function of radius is expressed by a simple powerlaw. The disk has a total mass of $0.01 M_{\odot}$ and extends from 0.07 au (the dust sublimation radius) to 620 au where the total vertical hydrogen column density reaches $N_{\langle\mathrm{H}\rangle \text {,ver }} \approx 10^{20} \mathrm{~cm}^{-2}$ (panel (f) in Fig. 4). 

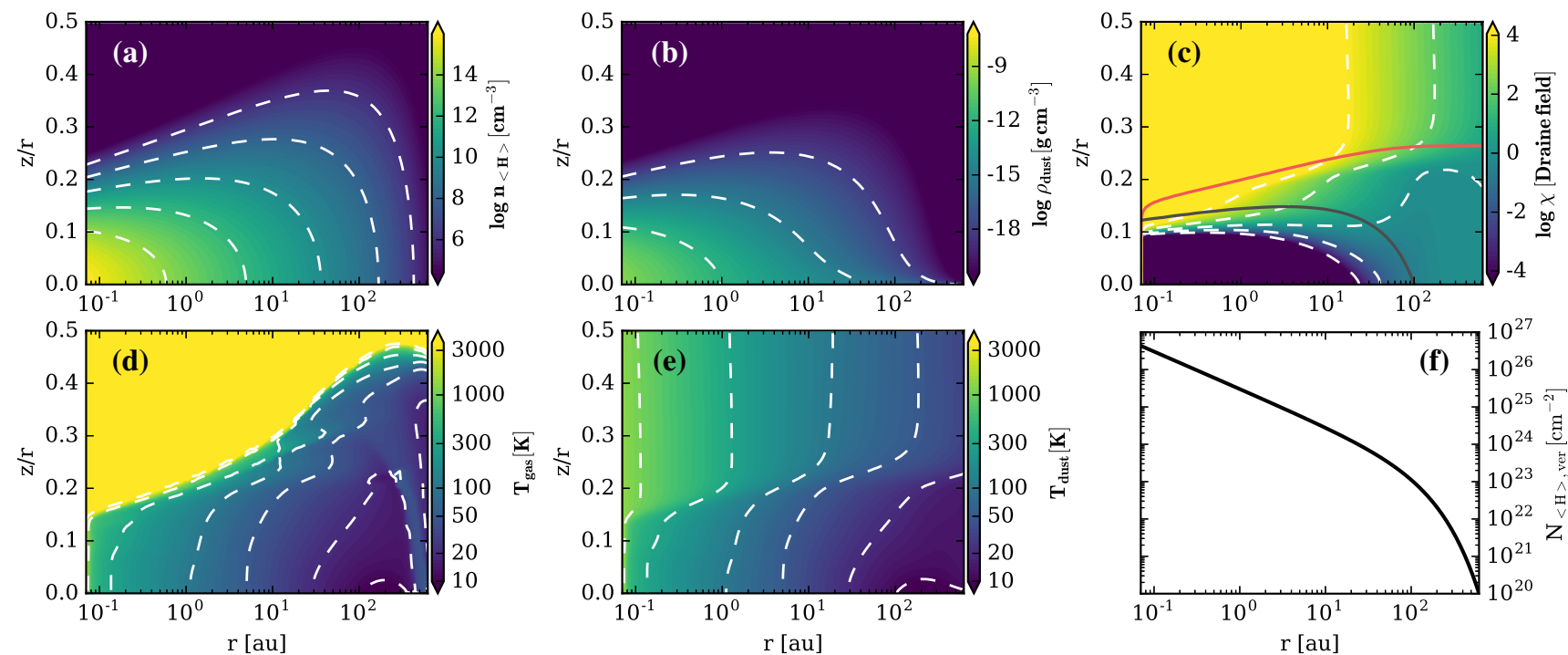

Fig. 4. Two dimensional structure of the reference disk model CI_XN. The height of the disk $z$ is scaled by the radius $(z / r)$. From top left to the bottom right: a) gas number density $n_{\langle\mathrm{H}\rangle}$; b) dust density $\rho_{\text {dust }}$ (note the dust settling); c) FUV radiation field $\chi$ in units of the ISM Draine field; d) gas temperature $\left.T_{\text {gas }} ; \mathbf{e}\right)$ dust temperature $T_{\text {dust }}$ and f) the vertical hydrogen column density $N_{\langle\mathrm{H}\rangle \text {,ver }}$ versus radius. The white dashed contour lines in each contour plot correspond to the levels shown in the respective colorbar. The black (red) solid contour in panel c) indicate a vertical (radial) visual extinction equal to unity.

Table 4. Main fixed parameters of the disk model.

\begin{tabular}{|c|c|c|}
\hline Quantity & Symbol & Value \\
\hline stellar mass & $M_{*}$ & $0.7 M_{\odot}$ \\
\hline stellar effective temp. & $T_{*}$ & $4000 \mathrm{~K}$ \\
\hline stellar luminosity & $L_{*}$ & $1.0 L_{\odot}$ \\
\hline FUV excess & $L_{\mathrm{FUV}} / L_{*}$ & 0.01 \\
\hline FUV power law index & $p_{\mathrm{UV}}$ & 1.3 \\
\hline strength of interst. FUV ${ }^{a}$ & $\chi^{\mathrm{ISM}}$ & 1 \\
\hline disk gas mass & $M_{\text {disk }}$ & $0.01 M_{\odot}$ \\
\hline dust/gas mass ratio & $d / g$ & 0.01 \\
\hline inner disk radius & $R_{\text {in }}$ & $0.07 \mathrm{au}$ \\
\hline tapering-off radius & $R_{\text {tap }}$ & $100 \mathrm{au}$ \\
\hline column density power ind. & $\gamma$ & 1.0 \\
\hline reference scale height & $H(100 \mathrm{au})$ & $10 \mathrm{au}$ \\
\hline flaring power index & $\beta$ & 1.15 \\
\hline \multirow{8}{*}{$\begin{array}{l}\text { min. dust particle radius } \\
\text { max. dust particle radius } \\
\text { dust size dist. power index } \\
\text { turbulent mixing param. } \\
\text { max. hollow volume ratio } \\
\text { dust composition } \\
\text { (volume fractions) }\end{array}$} & $a_{\min }$ & $0.05 \mu \mathrm{m}$ \\
\hline & $a_{\max }$ & $3 \mathrm{~mm}$ \\
\hline & $a_{\text {pow }}$ & 3.5 \\
\hline & $\alpha_{\text {settle }}$ & $10^{-2}$ \\
\hline & $V_{\text {hollow,max }}$ & 0.8 \\
\hline & $\mathrm{Mg}_{0.7} \mathrm{Fe}_{0.3} \mathrm{SiO}_{3}$ & $60 \%$ \\
\hline & amorph. carbon & $15 \%$ \\
\hline & porosity & $25 \%$ \\
\hline
\end{tabular}

Notes. If not noted otherwise, these parameters are kept fixed for all our models presented in this work. For more details on the parameter definitions see Woitke et al. $(2009,2011,2016) .{ }^{(a)} \chi^{\mathrm{ISM}}$ is given in units of the Draine field (Draine \& Bertoldi 1996; Woitke et al. 2009).

(b) We use distributed hollow spheres for the dust opacity calculations (Min et al. 2005, 2016).

For the dust density distribution we assumed a dust to gas mass ratio of $d / g=0.01$. There is observational evidence for dust growth and settling in protoplanetary disks (e.g. Williams \& Cieza 2011; Dullemond \& Dominik 2004). To account for dust growth we assumed a power law dust size distribution $f(a)=a^{-3.5}$ with a minimum and maximum grain radius of $a_{\min }=0.05 \mu \mathrm{m}$ and $a_{\max }=3000 \mu \mathrm{m}$. For dust settling we applied the method of Dubrulle et al. (1995) with a turbulent mixing parameter of $10^{-2}$.

The irradiation of the disk by the star is important for the temperature and the chemical composition of the disk. For the photospheric emission of the star we use PHOENIX stellar atmosphere models (Brott \& Hauschildt 2005). We considered a $0.7 M_{\odot}$ star with an effective temperature of $4000 \mathrm{~K}$ and a luminosity of $1 L_{\odot}$. In addition to the photospheric emission, T Tauri stars commonly show far ultra-violet (FUV) excess (e.g. France et al. 2014) due to accretion shocks and strong X-ray emission (e.g. Güdel \& Nazé 2009). For the excess FUV emission, we used a simple power law spectrum with a total integrated FUV luminosity of $L_{\mathrm{FUV}}=0.01 L_{*}$, in the wavelength interval $[91.2 \mathrm{~nm}, 250 \mathrm{~nm}]$. The details for the stellar X-ray properties are discussed in Sect. 2.3.1 above.

We use the molecules $\mathrm{HCO}^{+}$and $\mathrm{N}_{2} \mathrm{H}^{+}$primarily to study the impact of SP ionization. To verify if our model gives reasonable results concerning $\mathrm{HCO}^{+}$and $\mathrm{N}_{2} \mathrm{H}^{+}$abundances, we compared the modelled fluxes for the $J=3-2$ transition of $\mathrm{HCO}^{+}$ and $\mathrm{N}_{2} \mathrm{H}^{+}$with the observational sample of Öberg et al. (2010, 2011a) finding a good agreement (for details see Appendix E).

\subsection{Model series}

It is likely that the different ionization sources are correlated. As already discussed the SP flux of young stars is actually derived from their stellar X-ray properties (Lee et al. 1998b; Feigelson et al. 2002). Also the CR ionization rate might be anti-correlated with the activity of the star (Cleeves et al. 2013). However, these possible correlations are not well understood. We therefore ran a series of full disk models using the $\mathrm{X}$-ray, SP and CR spectra (described above) as inputs and also included the Turner SP model (Appendix D). We do not discuss any model with the active Sun SP spectrum as in this case SPs do not have a significant impact on the disk chemical structure 
Table 5. Model series.

\begin{tabular}{llll}
\hline \hline Name & X-rays & Stellar particles & Cosmic rays \\
\hline CI_XN & normal $^{a}$ & - & ISM $^{d}$ \\
CI_XH & high $^{b}$ & - & ISM \\
CI_XN_SP & normal $^{-}$ & active T Tauri & ISM \\
CI_XH_SP & high & active T Tauri & ISM \\
CI_T & Turner & Turner & ISM \\
CL_XN & normal & - & low \\
CL_XH & high & - & low \\
CL_XN_SP & normal & active T Tauri & low \\
CL_XH_SP & high & active T Tauri & low \\
CL_T & Turner & Turner & low \\
\hline
\end{tabular}

Notes. In the model names CI (CL) stands for ISM (low) CR ionization rates, XN (XH) for normal (high) X-ray luminosities, SP for stellar particles and $\mathrm{T}$ for Turner model. ${ }^{(a)} \mathrm{X}$-ray luminosity $L_{\mathrm{X}}=10^{30} \mathrm{erg} \mathrm{s}^{-1}$. (b) $L_{\mathrm{X}}=5 \times 10^{30} \mathrm{erg} \mathrm{s}^{-1}$. (c) $L_{\mathrm{X}}=2 \times 10^{30} \mathrm{erg} \mathrm{s}^{-1}$. (d) $\mathrm{CR}$ ionization rate $\zeta_{\mathrm{CR}} \approx 2 \times 10^{-17} \mathrm{~s}^{-1}$. ${ }^{(e)} \zeta_{\mathrm{CR}} \approx 2 \times 10^{-19} \mathrm{~s}^{-1}$.

(see Sect. 3.1). An overview of all presented models is given in Table 5.

\section{Results}

\subsection{Ionization rates as a function of column density}

Before we discuss our results for the full disk model, we compare the SP, X-ray and CR ionization rates as a function of the total hydrogen column density $N_{\langle\mathrm{H}\rangle}\left(N_{\langle\mathrm{H}\rangle}=N_{\mathrm{H}}+2 N_{\mathrm{H}_{2}}\right)$. Figure 5 shows such a comparison for our different input spectra discussed in Sects. 2.2 and 2.3.

From visual inspection of Fig. 5 it becomes clear that for a SP flux comparable to our Sun (active Sun spectrum) SP ionization cannot compete with X-ray ionization assuming typical T Tauri $\mathrm{X}$-ray luminosities. However, for the active T Tauri SP spectrum SP ionization becomes comparable to X-ray ionization or even dominates for $N_{\langle\mathrm{H}\rangle} \lesssim 10^{24}-10^{25} \mathrm{~cm}^{-2}$. For $N_{\mathrm{H}} \lesssim 10^{23} \mathrm{~cm}^{-2} \zeta_{\mathrm{SP}}$ is determined by the particles with $E_{\mathrm{p}} \lesssim 5 \times 10^{7} \mathrm{eV}$ whereas higher energy particles dominate for $N_{\langle\mathrm{H}\rangle} \gtrsim 10^{23} \mathrm{~cm}^{-2}$. The kink at $N_{\langle\mathrm{H}\rangle} \approx 2 \times 10^{25} \mathrm{~cm}^{-2}$ is caused by the rapid attenuation of the SPs at high column densities. At such high column densities even the most energetic particles have lost most of their energy and the ionization rate drops exponentially.

For X-rays, Fig. 5 shows the differences between the normal and high X-ray spectrum. The X-ray ionization rates are higher for the high X-ray spectrum due to the higher X-ray luminosity. Additionally, the harder X-ray photons can penetrate to deeper layers but are also more efficiently scattered than lower energy X-ray photons. Compared to the normal X-ray case, the $\mathrm{X}$-ray ionization rate increases by several orders of magnitude for $N_{\langle\mathrm{H}\rangle} \gtrsim 10^{24} \mathrm{~cm}^{-2}$ for the high X-ray case.

Galactic cosmic rays are the most energetic ionization source. The peak in the particle energy distribution is around $10^{8}-10^{9} \mathrm{eV}$ (see Fig. 2). As a consequence the CR ionization rate $\zeta_{\mathrm{CR}}$ stays mostly constant and only decrease for $N_{\langle\mathrm{H}\rangle} \gtrsim 10^{25} \mathrm{~cm}^{-2}$. Only for such high column densities CR particle absorption becomes efficient.

In the Turner model it is implicitly assumed that SPs have the same energy distribution than Galactic CRs (see Appendix D). As a consequence the SP ionization rate in the Turner model is simply a scaled up version of the $\mathrm{CR}$ ionization rate. The slight differences to our model in CR attenuation is caused by the

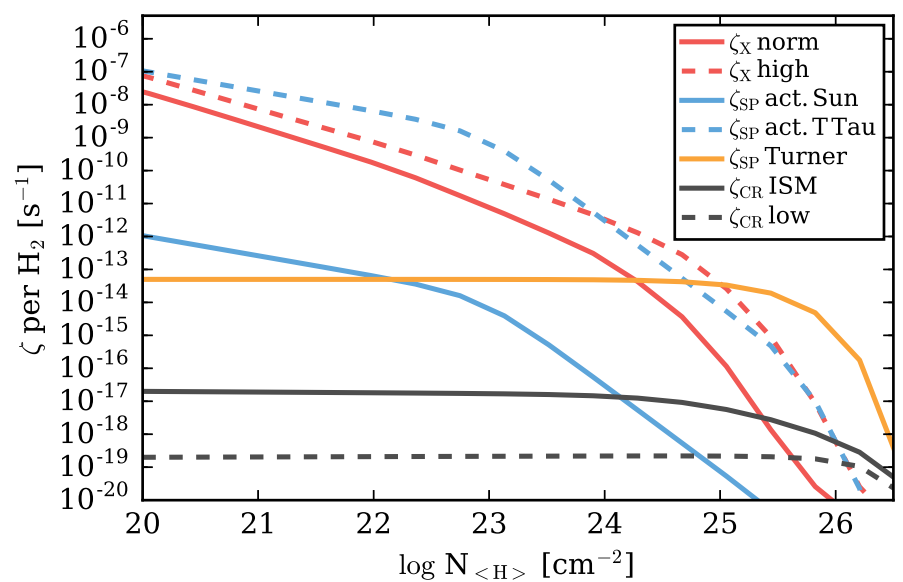

Fig. 5. SP, CR and X-ray ionization rates $\zeta$ as a function of hydrogen column density $N_{\langle\mathrm{H}\rangle}$.

different methods used to calculate the SP/CR ionization rates; Turner \& Drake (2009) use the fitting formulae of Umebayashi \& Nakano (2009). Compared to our active T Tauri SP spectrum $\zeta_{\mathrm{SP}}$ in the Turner model is larger for $N_{\langle\mathrm{H}\rangle}>10^{25} \mathrm{~cm}^{-2}$ but significantly lower at low column densities.

\subsection{Disk ionization rates}

In Fig. 6 we show the ionization rates as a function of the vertical hydrogen column density $N_{\langle\mathrm{H}\rangle \text {,ver }}$ at two different radii of the disk. Shown on the figure are the models CI_XN_SP (ISM CR, normal X-rays; left panel) and CL_XH_SP (low CR, high $\mathrm{X}$-rays; right panel).

CRs are only significantly attenuated for $N_{\langle\mathrm{H}\rangle, \text { ver }}>10^{25} \mathrm{~cm}^{-2}$ and $r \lesssim 1$ au. For most of the disk, CRs provide a nearly constant ionization rate of $\zeta_{\mathrm{CR}} \approx 2 \times 10^{-17} \mathrm{~s}^{-1}$ for the ISM like and $\zeta_{\mathrm{CR}} \approx$ $2 \times 10^{-19} \mathrm{~s}^{-1}$ for the low CR spectrum.

$\mathrm{X}$-rays are strongly attenuated as a function of height and radius (i.e. geometric dilution). However, due to scattering X-rays can become the dominant midplane ionization source for large regions of the disk. For ISM-like CRs, CR ionization is the dominant midplane ionization source even in the high X-ray models. In the low CRs models X-rays are the dominant midplane ionization source for $r \lesssim 100$ au in the normal X-ray model and for all radii in the high X-ray model.

Differently to X-rays, SPs are not scattered towards the midplane. Due to their high energies they propagate along straight lines (provided that the SPs are not shielded by magnetic fields, see Sect. 4.3.2). As SPs are of stellar origin they penetrate the disk only along radial rays. The radial column densities close to the midplane of the disk are $N_{\langle\mathrm{H}\rangle \text {,rad }} \gg 10^{25} \mathrm{~cm}^{-2}$ and therefore SPs are already strongly attenuated at the inner rim of the disk. From Fig. 6 we see that the SP ionization rate $\zeta_{\text {SP }}$ drops below $10^{-19} \mathrm{~s}^{-1}$ for $N_{\langle\mathrm{H}\rangle \text {,ver }} \gtrsim 10^{24} \mathrm{~cm}^{-2}$ at $r=1 \mathrm{au}$ and for $N_{\langle\mathrm{H}\rangle \text {,ver }} \gtrsim 10^{22} \mathrm{~cm}^{-2}$ at $r=100$ au. However, at higher layers $\left(N_{\langle\mathrm{H}\rangle \text {,ver }} \lesssim 10^{22}-10^{23} \mathrm{~cm}^{-2}\right)$ SPs are the dominant ionization source even in the high X-ray models. Expressed in radial column densities: SPs are the dominant ionization source in disk regions with $N_{\langle\mathrm{H}\rangle \text {,rad }} \lesssim 10^{24}-10^{25} \mathrm{~cm}^{-2}$.

In Fig. 7 we show the dominant ionization source at every point in the disk for all SP models. An ionization source is dominant at a certain point in the disk if its value is higher than the sum of the two other ionization sources. The first two columns in Fig. 7 show our models, the last column shows the Turner models. In our models, SPs are the dominant ionization source in the 

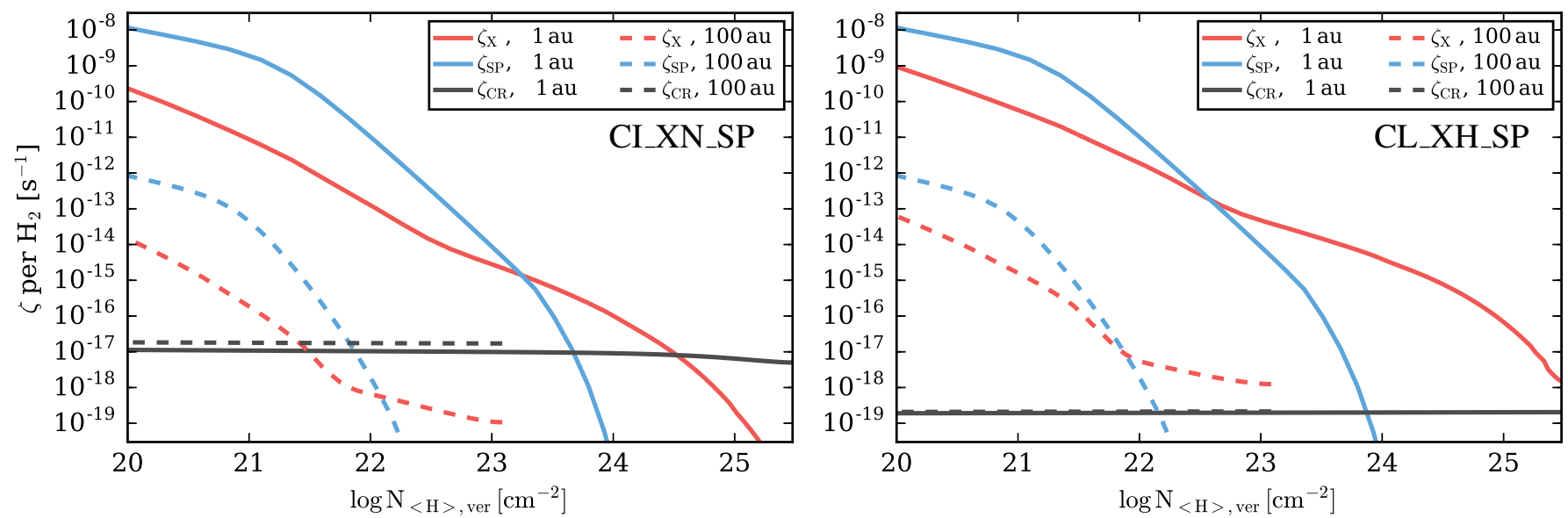

Fig. 6. Ionization rates $\zeta$ as a function of vertical column density $N_{\langle\mathrm{H}\rangle \text {,ver }}$ at radii of 1 and 100 au (solid and dashed lines respectively). The maximum values for $N_{\langle\mathrm{H}\rangle \text {,ver }}$ at the midplane of the disk, are $N_{\langle\mathrm{H}\rangle \text {,ver }} \approx 4 \times 10^{25} \mathrm{~cm}^{-2}$ and $N_{\langle\mathrm{H}\rangle, \text { ver }} \approx 2 \times 10^{23} \mathrm{~cm}^{-2}$ at 1 au and 100 au, respectively. Red lines are for X-rays, blue lines are for SPs and the black lines are for CRs. Left panel: model CI_XN_SP with ISM CRs and normal X-rays; right panel: model CL_XH_SP with low CRs and high X-rays.
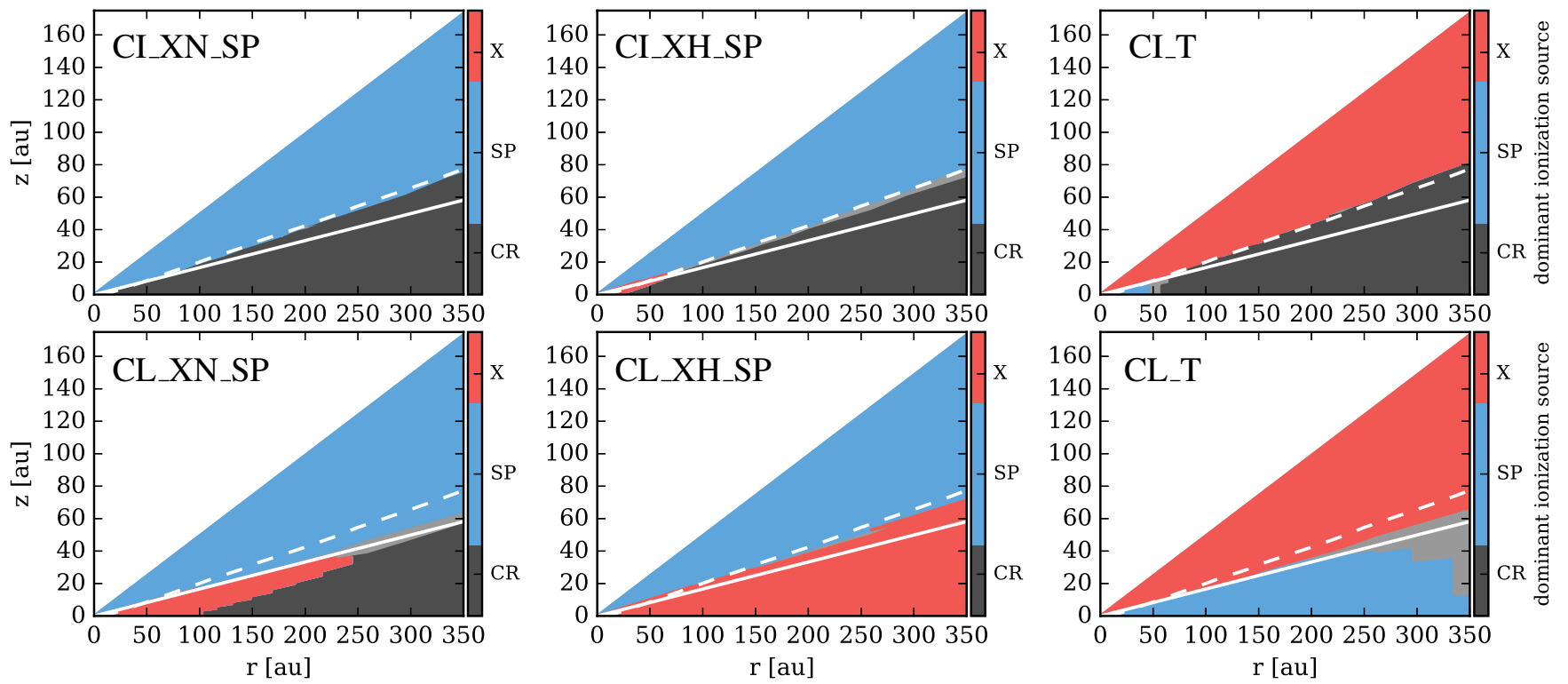

Fig. 7. Dominant disk ionization source throughout the disk. A light grey area indicates a region without a dominant ionization source. The different possible ionization sources, X-rays, SPs and CRs are identified by the different colors (color bar). The white solid contour line shows $N_{\langle\mathrm{H}\rangle \text {,rad }}=10^{25} \mathrm{~cm}^{-2}$ the white dashed line shows the CO ice line. The model names are given in the top left of each panel. Top row: models with ISM CR ionization rate (CI); bottom row: models with low CR ionization rate (CL). First column: normal X-ray models (XN); second column: high X-ray models $(\mathrm{XH})$; third column: turner models (T).

upper layers of the disk (above the white solid contour line for $N_{\langle\mathrm{H}\rangle \text {,rad }}=10^{25} \mathrm{~cm}^{-2}$ ), whereas in the midplane always CRs or $\mathrm{X}$-rays dominate.

For the Turner model the picture is quite different. In their model SPs can also penetrate the disk vertically (Appendix D) and reach higher vertical column densities before they are completely attenuated (Fig. 5). As a consequence SPs can become the dominant ionization source in the midplane of the disk (e.g. for the low $\mathrm{CR}$ case). In the upper layers always X-rays dominate as $\zeta_{\mathrm{SP}}<\zeta_{\mathrm{X}}$ for low column densities. In the Turner model $\zeta_{\mathrm{SP}} \lesssim 10^{-13} \mathrm{~s}^{-1}$ for $N_{\langle\mathrm{H}\rangle \text {,rad }}<10^{25} \mathrm{~cm}^{-2}$ which is several orders of magnitudes lower than in our models. The reason for this is that in the Turner model SPs are simply a scaled version of ISM like CRs. The high $\zeta_{\text {SP }}$ values in our model in the upper layers of the disk are caused by the high number of particles with energies $E_{\mathrm{p}} \lesssim 10^{8} \mathrm{eV}$, which are missing in the Turner model.

\subsection{Impact on $\mathrm{HCO}^{+}$and $\mathrm{N}_{2} \mathrm{H}^{+}$}

The molecules $\mathrm{HCO}^{+}$and $\mathrm{N}_{2} \mathrm{H}^{+}$are the two most observed molecular ions in disks (e.g. Dutrey et al. 2014) and are commonly used to trace the ionization structure of disks (e.g. Dutrey et al. 2007; Öberg et al. 2011b; Cleeves et al. 2015). Also Ceccarelli et al. (2014) used these two molecules to trace SPs in a protostellar envelope.

The main formation path of $\mathrm{HCO}^{+}$and $\mathrm{N}_{2} \mathrm{H}^{+}$is the ionneutral reaction of $\mathrm{H}_{3}^{+}$with their parent molecules $\mathrm{CO}$ and $\mathrm{N}_{2}$, respectively. $\mathrm{H}_{3}^{+}$is created by ionization of $\mathrm{H}_{2}$ by CRs, $\mathrm{X}$-rays and in our model additionally by SPs. The main destruction pathway for $\mathrm{HCO}^{+}$and $\mathrm{N}_{2} \mathrm{H}^{+}$is via dissociative recombination with free electrons.

The chemistry of $\mathrm{HCO}^{+}$and $\mathrm{N}_{2} \mathrm{H}^{+}$is linked to the $\mathrm{CO}$ freeze-out. To form $\mathrm{HCO}^{+}$, gas phase $\mathrm{CO}$ is required, whereas 

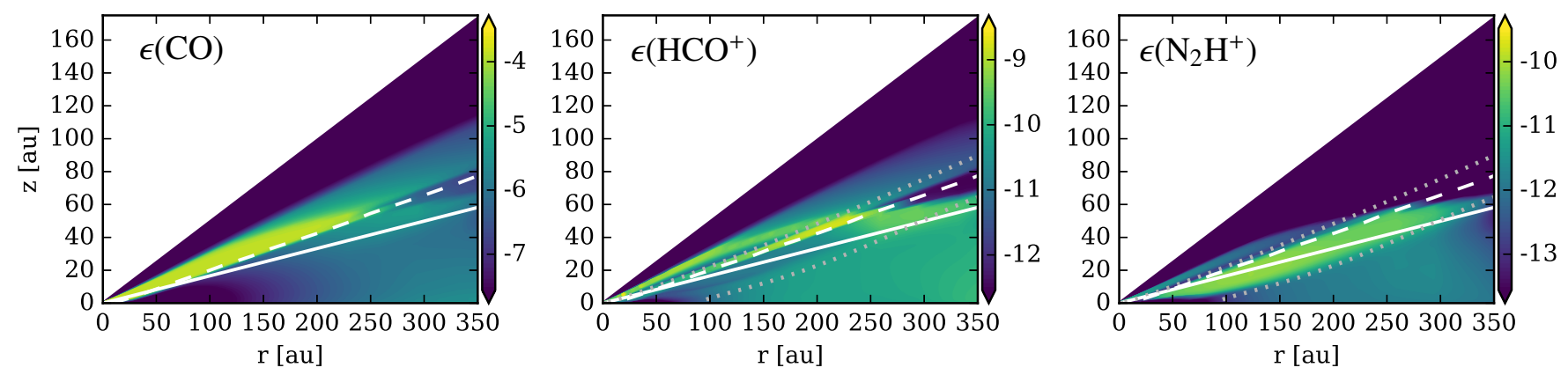

Fig. 8. Abundances $\epsilon(X)$ relative to hydrogen for $\mathrm{CO}, \mathrm{HCO}^{+}$and $\mathrm{N}_{2} \mathrm{H}^{+}$for the reference model CI_XN. The white solid contour line shows $N_{\langle\mathrm{H}\rangle \text {,rad }}=10^{25} \mathrm{~cm}^{-2}$, the white dashed line shows the CO ice line. We call the regions above and below the CO ice line the warm and cold molecular layer, respectively. The dotted iso-contours show where the X-ray ionization rate is equal to the ISM CR $\left(\zeta_{\mathrm{CR}}=2 \times 10^{-17} \mathrm{~s}^{-1}\right)$ and equal to the low $\mathrm{CR}\left(\zeta_{\mathrm{CR}}=2 \times 10^{-19} \mathrm{~s}^{-1}\right)$ ionization rate, respectively.

$\mathrm{N}_{2} \mathrm{H}^{+}$is efficiently destroyed by CO (e.g. Aikawa et al. 2015). Consequently the $\mathrm{N}_{2} \mathrm{H}^{+}$abundance peaks in regions where $\mathrm{CO}$ is depleted and $\mathrm{N}_{2}$, the precursor of $\mathrm{N}_{2} \mathrm{H}^{+}$, is still in the gas phase. The result of this chemical interaction is a vertically layered chemical structure for $\mathrm{HCO}^{+}$and $\mathrm{N}_{2} \mathrm{H}^{+}$(see Fig. 8). For further details on the $\mathrm{HCO}^{+}$and $\mathrm{N}_{2} \mathrm{H}^{+}$chemistry see Appendix $\mathrm{B}$, where we also list the main formation and destruction pathways for $\mathrm{HCO}^{+}$and $\mathrm{N}_{2} \mathrm{H}^{+}$(Table B.1).

\subsubsection{Abundance structure}

In the following we describe details of the molecular abundance structure that are relevant for the presentation of our results for our reference model CI_XN. The abundance $\epsilon$ of a molecule X is given by $\epsilon(\mathrm{X})=n_{\mathrm{X}} / n_{\langle\mathrm{H}\rangle}$, where $n_{\mathrm{X}}$ is the number density of the respective molecule and $n_{\langle\mathrm{H}\rangle}=n_{\mathrm{H}}+2 n_{\mathrm{H}_{2}}$ is the total hydrogen number density. Figure 8 shows the resulting abundance structure for $\mathrm{CO}, \mathrm{HCO}^{+}$and $\mathrm{N}_{2} \mathrm{H}^{+}$for the $\mathrm{CI} \_\mathrm{XN}$ model.

We define the location of the $\mathrm{CO}$ ice line where the $\mathrm{CO}$ gas phase abundance is equal to the $\mathrm{CO}$ ice-phase abundance (white dashed line in Fig. 8). The $\mathrm{CO}$ ice line is located at dust temperatures in the range $T_{\mathrm{d}} \approx 23-32 \mathrm{~K}$ (density dependence of the adsorption/desorption equilibrium; e.g. Furuya \& Aikawa 2014). The radial CO ice line in the midplane $(z=0 \mathrm{au})$ is at $r \approx 12$ au and $T_{\mathrm{d}} \approx 32 \mathrm{~K}$. At $r \approx 50$ au the vertical CO ice line is at $z \approx 8.5 \mathrm{au}(z / r \approx 0.17)$ and $T_{\mathrm{d}} \approx 26 \mathrm{~K}$. Inside/above the CO ice line $\epsilon(\mathrm{CO}) \approx 10^{-4}$. Outside/below the CO ice line $\epsilon(\mathrm{CO})$ rapidly drops to values $\lesssim 10^{-6}$. In regions where non-thermal desorption processes are efficient $(r \gtrsim 150 \mathrm{au}) \epsilon(\mathrm{CO}) \approx 10^{-6}$ down to the midplane. For the regions inside/above and outside/below the $\mathrm{CO}$ ice line we use the terms warm and cold molecular layer, respectively.

There are two main reservoirs for $\mathrm{HCO}^{+}$, one in the warm molecular layer above the $\mathrm{CO}$ ice line and one in the outer disk ( $r \gtrsim 150 \mathrm{au}$ ) below the CO ice line where non-thermal desorption becomes efficient. In the warm molecular layer, the ionization fraction $\epsilon\left(\mathrm{e}^{-}\right) \approx 10^{-7}$ is dominated by sulphur as it is ionized by UV radiation (e.g. Teague et al. 2015, see also Sect. 4.2.3). Those free electrons efficiently destroy molecular ions via dissociative recombination. This causes a dip in the vertical $\mathrm{HCO}^{+}$abundance structure within the warm molecular layer with $\epsilon\left(\mathrm{HCO}^{+}\right) \approx 10^{-12}-10^{-11}$, whereas at the top and the bottom of the warm molecular layer $\epsilon\left(\mathrm{HCO}^{+}\right)$reaches values of $\approx 10^{-10}-10^{-9}$. The peak in the top layer is mainly caused by the high $\mathrm{X}$-ray ionization rate for $\mathrm{H}_{2}\left(\zeta_{\mathrm{X}} \gtrsim 10^{-12} \mathrm{~s}^{-1}\right)$. At the bottom of the warm molecular layer more $\mathrm{HCO}^{+}$survives. This region is already sufficiently shielded from UV radiation and the free electron abundance drops rapidly. In the second reservoir, below the $\mathrm{CO}$ ice line where non-thermal desorption is efficient $\epsilon\left(\mathrm{HCO}^{+}\right) \approx 10^{-11}-10^{-10}$.

The main $\mathrm{N}_{2} \mathrm{H}^{+}$reservoir resides in the cold molecular layer just below the $\mathrm{CO}$ ice line with $\epsilon\left(\mathrm{N}_{2} \mathrm{H}^{+}\right) \gtrsim 10^{-11}$. The lower boundary of this layer with $\epsilon\left(\mathrm{N}_{2} \mathrm{H}^{+}\right)<10^{-11}$ is reached at $T_{\mathrm{d}} \approx 16 \mathrm{~K}$ where $\epsilon\left(\mathrm{N}_{2}\right) \lesssim 10^{-6}$ due to freeze-out. Radially this layer extends from the inner midplane $\mathrm{CO}$ ice line out to $r \approx 250-300 \mathrm{au}$. Close to the midplane $\epsilon\left(\mathrm{N}_{2} \mathrm{H}^{+}\right) \lesssim 10^{-12}$ for $r \gtrsim 150$ au due to non-thermal desorption of ices. There is also a thin $\mathrm{N}_{2} \mathrm{H}^{+}$layer at the top of the warm molecular layer with $\epsilon\left(\mathrm{N}_{2} \mathrm{H}^{+}\right) \approx 10^{-12}$ extending from the inner radius of the disk out to $r \approx 100 \mathrm{au}$. In this layer the $\mathrm{X}$-ray ionization rate is high enough to compensate for the destruction of $\mathrm{N}_{2} \mathrm{H}^{+}$by $\mathrm{CO}$.

The detailed appearance of this layered structure is especially sensitive to the dust temperature and therefore also to dust properties (e.g. dust size distribution). The above described abundance structure for $\mathrm{CO}, \mathrm{HCO}^{+}$and $\mathrm{N}_{2} \mathrm{H}^{+}$is consistent with the model of Aikawa et al. (2015) that includes millimetre sized dust particles with a dust size distribution similar to what is used here (for details see Appendix B).

\subsubsection{Vertical column densities}

To study the impact of SP ionization quantitatively we compare vertical column densities of $\mathrm{HCO}^{+}$and $\mathrm{N}_{2} \mathrm{H}^{+}$for models with and without SPs. In Fig. 9 we show the vertical column densities $N_{\mathrm{HCO}^{+}}$and $N_{\mathrm{N}_{2} \mathrm{H}^{+}}$as a function of the disk radius $r$ for all models listed in Table 5. The left column in Fig. 9 shows the models with ISM CRs, the right column the models with low CRs. At first we discuss the models without SPs and compare them to other theoretical models.

The $N_{\mathrm{HCO}^{+}}$profile shows a dip around $r \approx 50-100$ au in the ISM CR models CI_XN and CI_XH (high X-rays). This dip is also seen in the models of Cleeves et al. (2014). Unlike Cleeves et al. (2014), in our model this dip is not predominantly due to the erosion of $\mathrm{CO}$ by reactions with $\mathrm{He}^{+}$("sink effect" e.g. Aikawa et al. 1997, 2015; Bergin et al. 2014; Furuya \& Aikawa 2014), but mainly due to the interplay of CO freeze-out and non-thermal desorption in the outer disk. The CO sink effect is also active in our model but less efficient (see Appendix B.2.2).

The lack of $\mathrm{HCO}^{+}$in the disk midplane at $r \approx 50-100 \mathrm{au}$ due to $\mathrm{CO}$ freeze-out is also visible in the $\mathrm{HCO}^{+}$abundance structure shown in Fig. 8. Non-thermal desorption in the midplane produces $\mathrm{CO}$ abundances $\gtrsim 10^{-7}$ for $r \gtrsim 150$ au and consequently also a slight increase in $\mathrm{N}_{\mathrm{HCO}^{+}}$. In the low CR models the ionization rate is too low to produce a significant amount 

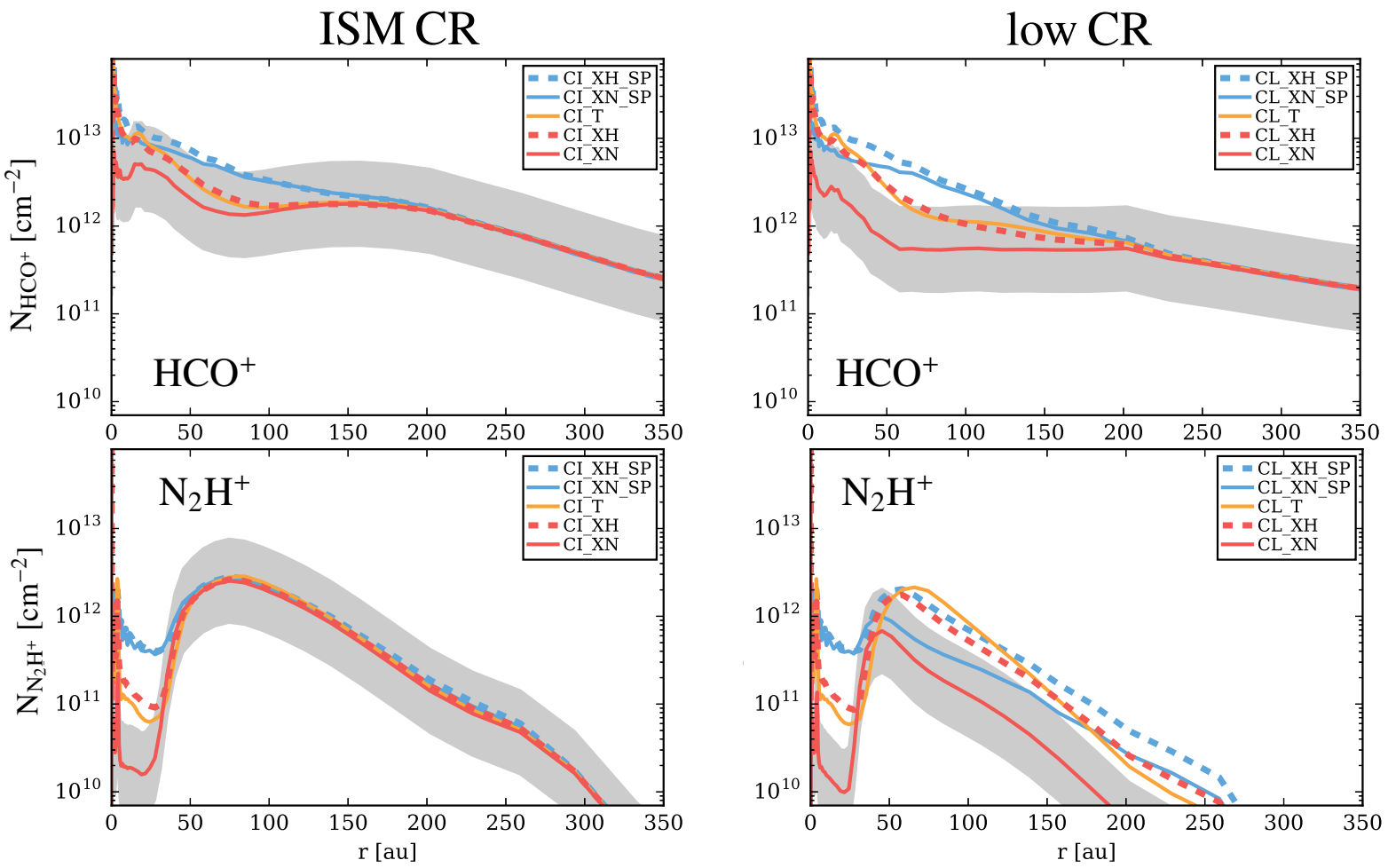

Fig. 9. Vertical column density profiles for $\mathrm{HCO}^{+}$and $\mathrm{N}_{2} \mathrm{H}^{+}$for our model series (Table 5). The left column shows the models with the ISM like CRs $\left(\zeta_{\mathrm{CR}} \approx 2 \times 10^{-17} \mathrm{~s}^{-1}\right)$, the right column with low CRs $\left(\zeta_{\mathrm{CR}} \approx 2 \times 10^{-19} \mathrm{~s}^{-1}\right)$. The top row shows $\mathrm{HCO}^{+}$, the bottom row $\mathrm{N}_{2} \mathrm{H}^{+}$. The blue lines are for models with, the red lines are for models without SPs. Dashed (solid) lines are for models with high (normal) X-rays. The orange solid line shows the Turner model. The grey shaded area marks a difference of a factor 3 in the column densities relative to the CI_XN (ISM CR, normal $\mathrm{X}$-rays) and CL_XN model (low CR, normal X-rays), respectively.

of $\mathrm{HCO}^{+}$in the cold molecular layer and the dip in the profile vanishes.

$\mathrm{N}_{2} \mathrm{H}^{+}$traces the distribution of gas phase $\mathrm{CO}$ as it is efficiently destroyed by CO (Qi et al. 2013a,b, 2015). This is also seen in our model. The sharp transition in the $\mathrm{N}_{2} \mathrm{H}^{+}$column density at $r \approx 30$ au traces the onset of CO freeze-out (Fig. 9). We note, however, that the actual midplane CO ice-line is at $\approx 12 \mathrm{au}$ (see Appendix C for details). In the ISM CR models $N_{\mathrm{N}_{2} \mathrm{H}^{+}}$is dominated by $\mathrm{CR}$ ionization as $\mathrm{N}_{2} \mathrm{H}^{+}$mainly resides in the cold molecular layer. In the warm $\mathrm{N}_{2} \mathrm{H}^{+}$layer X-ray ionization dominates. However, due to the lower densities in the warm molecular layer, this layer only contributes significantly to the column density within the radial $\mathrm{CO}$ ice line, and the impact of X-rays is only visible there (compare models CI_XH and CI_XN in Fig. 9). The high $\mathrm{X}$-ray luminosity decreases the contrast between the peak of $N_{\mathrm{N}_{2} \mathrm{H}^{+}}$close to the radial CO ice line and $\mathrm{N}_{\mathrm{N}_{2} \mathrm{H}^{+}}$ inside the $\mathrm{CO}$ ice line by about a factor of five.

For the low CR case $N_{\mathrm{N}_{2} \mathrm{H}^{+}}$drops by more than an order of magnitude compared to the ISM CR case. Such a strong impact of CR ionization on $N_{\mathrm{N}_{2} \mathrm{H}^{+}}$is also reported by Aikawa et al. (2015) and Cleeves et al. (2014). Higher X-ray luminosities can compensate for low $\mathrm{CR}$ ionization only to some extent. In the high X-ray model CL_XH, $N_{\mathrm{N}_{2} \mathrm{H}+}$ is lower by a factor of five compared to the ISM CR models.

\subsubsection{Impact of SPs}

The column densities $N_{\mathrm{HCO}^{+}}$and $N_{\mathrm{N}_{2} \mathrm{H}^{+}}$for models with SPs are shown in Fig. 9 (blue solid and dashed lines). The solid orange line in Fig. 9 shows the results for the Turner model. We discuss the Turner model separately in Sect. 3.3.4. We define a change in the column densities by at least a factor of three compared to the reference model as significant. This is indicated by the grey area around the column density profiles of the reference models CI_XN and CL_XN.

To better quantify the impact of SPs compared to the competing $\mathrm{H}_{2}$ ionization sources, $\mathrm{X}$-rays and $\mathrm{CRs}$, we introduce the weighted column density

$N_{\zeta}(r)=\int_{0}^{\infty} n(r, z) \times f_{\zeta}(r, z) \mathrm{d} z\left[\mathrm{~cm}^{-2}\right]$.

$N_{\zeta}$ is the weighted column density for a particular $\mathrm{H}_{2}$ ionization source $\zeta, n$ is the number density of a particular molecule in units of $\mathrm{cm}^{-3}$ and

$f_{\zeta}=\frac{\zeta}{\zeta_{\mathrm{X}}+\zeta_{\mathrm{SP}}+\zeta_{\mathrm{CR}}}$.

$N_{\zeta}$ represents the fraction of the column density dominated by a particular ionization source $\zeta$.

In Fig. 10 we show $N_{\zeta}$ for $\mathrm{HCO}^{+}$and $\mathrm{N}_{2} \mathrm{H}^{+}$normalized to the total column density of the respective molecule as a function of radius. The region where more than $50 \%$ of the total column density of the molecules arise from disk regions above the $\mathrm{CO}$ ice line (i.e. from the warm molecular layer) is roughly indicated on each plot.

SP ionization has a significant impact on the $N_{\mathrm{HCO}^{+}}$profile in all our models. In the ISM CR model (CI_XN_SP) $N_{\mathrm{HCO}^{+}}$ increases by a factor $\approx 3$ for $50 \lesssim r \lesssim 100$ au and the dip in the profile seen in the models without SPs (CI_XN) vanishes (top left panel in Fig. 9). In the low CR models this region increases to $25 \lesssim r \lesssim 150$ au and $N_{\mathrm{HCO}^{+}}$reaches values up to an order of magnitude higher compared to the CL_XN model (top right panel in Fig. 9). In the models with high X-rays, SP ionization 

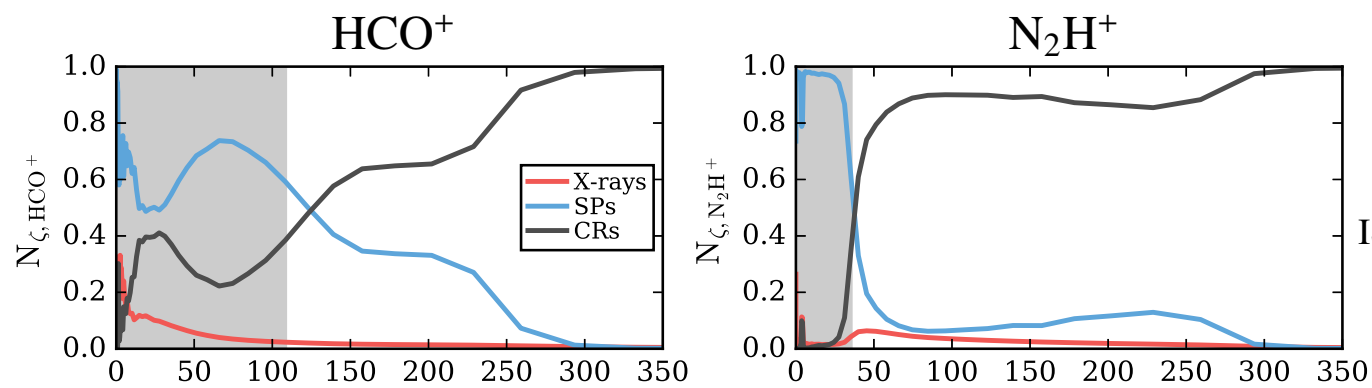

\section{CI_XN_SP}

ISM CR, normal X-rays
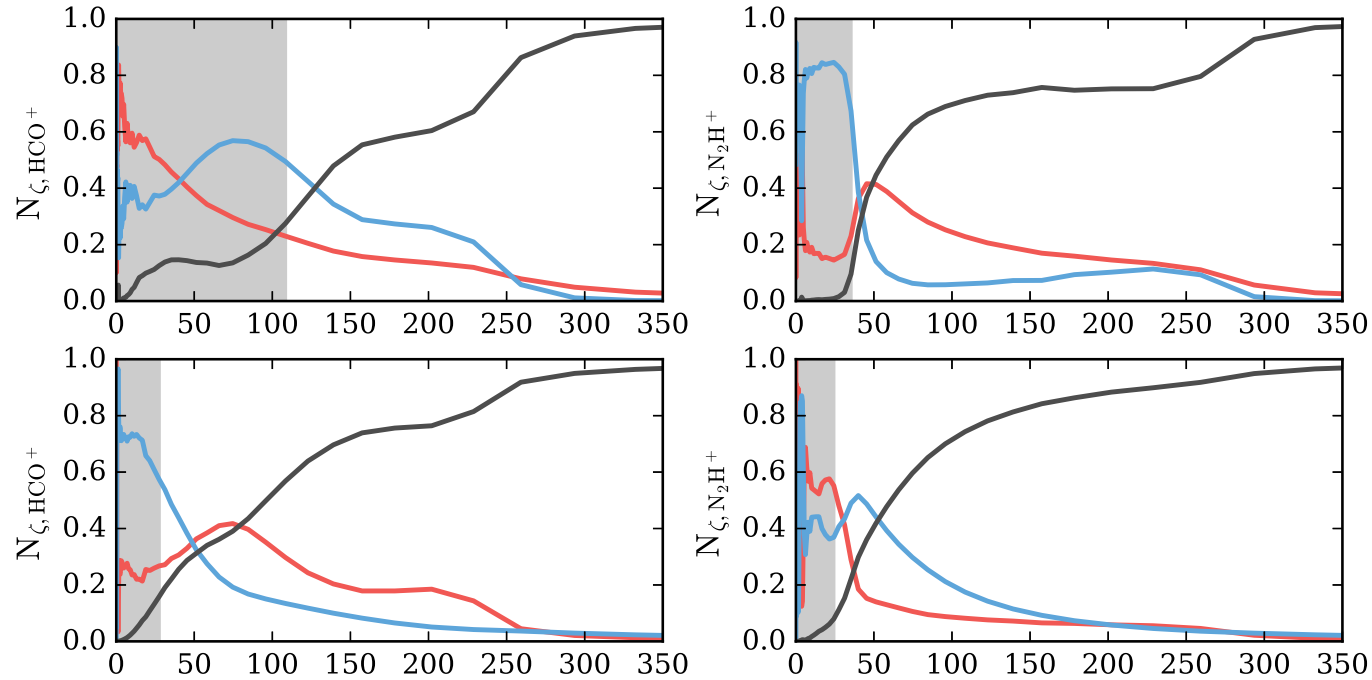

\section{CI_XH_SP}

ISM CR, high X-rays
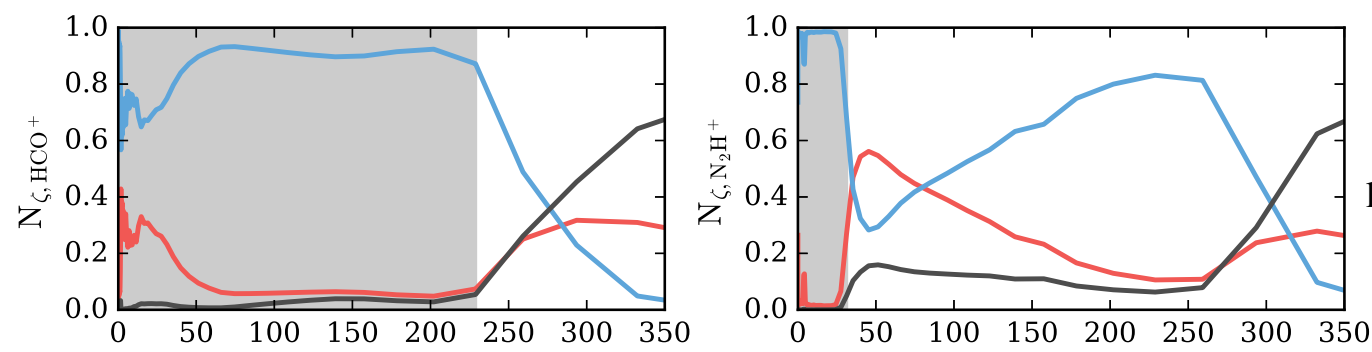

\section{CL_XN_SP}

low $\mathrm{CR}$, normal X-rays

\section{CI_T}

ISM CR, Turner
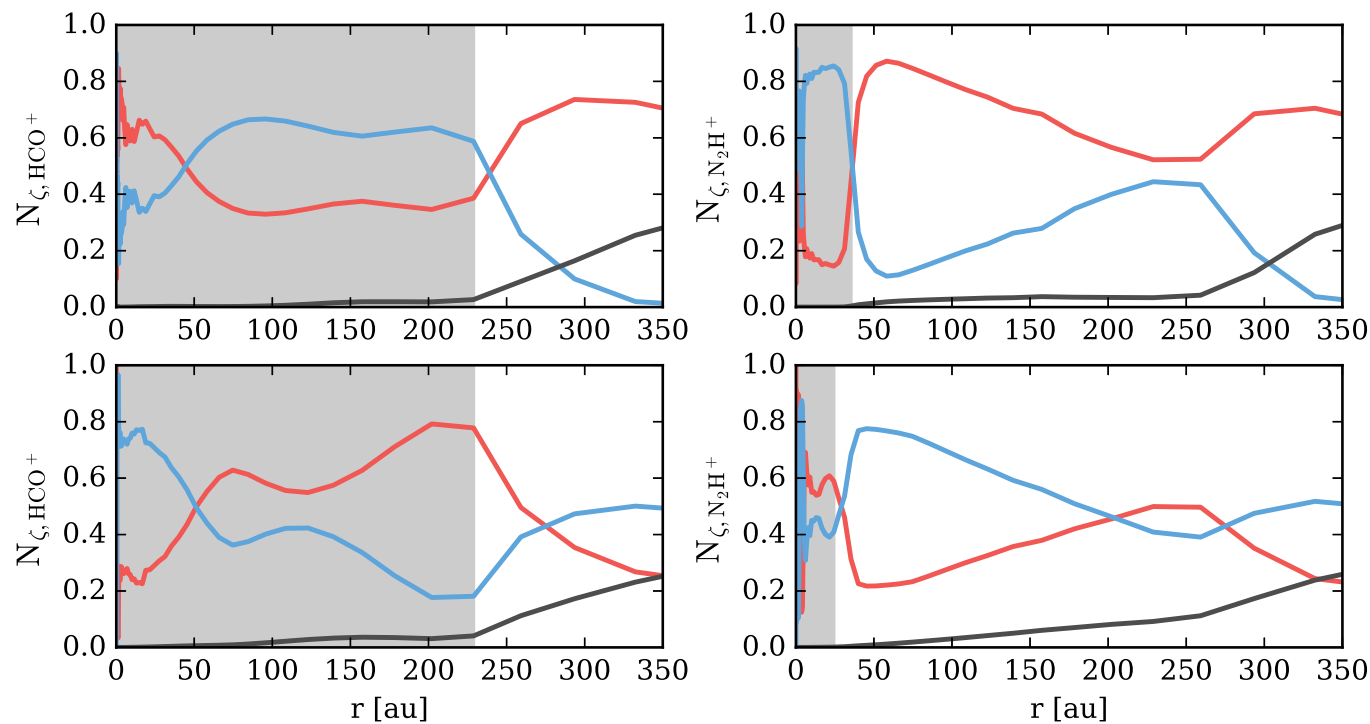

CL_XH_SP

low $\mathrm{CR}$, high $\mathrm{X}$-rays

Fig. 10. Ionization rate weighted column densities $\mathrm{N}_{\zeta}$ (Eq. (5)) as a function of radius for $\mathrm{HCO}^{+}$(left column) and $\mathrm{N}_{2} \mathrm{H}^{+}$(right column). $\mathrm{N}_{\zeta}$ is normalized to the total column density of the respective molecule. The individual colored solid lines show the fraction of the total column density dominated by a certain ionization source. Red is for X-rays $\left(\mathrm{N}_{\zeta \mathrm{X}}\right)$, blue for SPs $\left(\mathrm{N}_{\zeta_{\mathrm{SP}}}\right)$ and black for CRs $\left(\mathrm{N}_{\zeta \mathrm{CR}}\right)$. Each row corresponds to one model. On the right hand side the model descriptions are provided (see Table 5). The grey shaded area marks the region where more than 50\% of the column density arise from regions above the $\mathrm{CO}$ ice line (i.e. the warm molecular layer). 
still has an significant impact and $\mathrm{N}_{\mathrm{HCO}^{+}}$increases by up to a factor of three (dashed lines in Fig. 9).

This situation is also clearly visible in Fig. 10 where we show $N_{\zeta}$ as a function of radius (Eq. (5)). Although X-rays can be the dominant ionization source close to the star, in all models $N_{\mathrm{HCO}^{+}}$is dominated by SP ionization for $50 \lesssim r \lesssim 100-200 \mathrm{au}$. Figure 10 also shows that in the low CR models $N_{\mathrm{HCO}^{+}}$is mainly built up in the warm molecular layer for $r \lesssim 200 \mathrm{au}$. In this region (indicated in Fig. 10) the warm molecular layer contributes more to the total column density than the cold molecular layer.

For $\mathrm{N}_{2} \mathrm{H}^{+}$the picture is more complex. In the ISM CR models SPs have only very little impact on $\mathrm{N}_{\mathrm{N}_{2} \mathrm{H}^{+}}$. Only in the inner $30 \mathrm{au}$, within the radial $\mathrm{CO}$ ice line, the $\mathrm{N}_{2} \mathrm{H}^{+}$profile is significantly affected. The reason for this is the high SP ionization rate $\zeta_{\mathrm{SP}} \gtrsim 10^{-12} \mathrm{~s}^{-1}$ in the warm molecular layer of the disk close to the star. In this region the abundance ratio of $\mathrm{HCO}^{+} / \mathrm{N}_{2} \mathrm{H}^{+}$drops from $>10^{3}$, in the models without SPs, to around 10 to 100 in models with SPs. These high ratios can be explained by the efficient destruction of $\mathrm{N}_{2} \mathrm{H}^{+}$by CO. However, due to the high $\zeta_{\mathrm{SP}}$ this destruction path becomes less important, and the molecular ion abundance are mainly determined by the balance between ionization and recombination.

Ceccarelli et al. (2014) reported a very low measured $\mathrm{HCO}^{+} / \mathrm{N}_{2} \mathrm{H}^{+}$ratio between three and four in the Class 0 source OMC-2 FIR 4. They explain this low ratio by the high ionization rates due to SPs $\left(\zeta_{\mathrm{SP}}>10^{-14}\right)$. In our disk model $\zeta_{\mathrm{SP}}$ in the warm molecular layer is comparable, but the $\mathrm{HCO}^{+} / \mathrm{N}_{2} \mathrm{H}^{+}$ ratio is much larger than four. This higher ratio is due to the higher densities of $10^{8}-10^{10} \mathrm{~cm}^{-3}$ and the stronger UV field in the warm molecular layer, compared to the physical conditions in OMC-2 FIR 4. This is in agreement with the chemical models presented in Ceccarelli et al. (2014).

The impact of SPs on the $\mathrm{N}_{2} \mathrm{H}^{+}$abundance in the warm layer can extend out to $r \approx 200$ au (similar to $\mathrm{HCO}^{+}$). However, for $r>30$ au this layer does not significantly contribute to the total $\mathrm{N}_{2} \mathrm{H}^{+}$column density as $N_{\mathrm{N}_{2} \mathrm{H}^{+}}$is dominated by the high density layer below the vertical $\mathrm{CO}$ ice line.

Beyond the radial $\mathrm{CO}$ ice line $N_{\mathrm{N}_{2} \mathrm{H}^{+}}$is dominated by CRs in the ISM CR models and is not affected by X-rays nor SPs (bottom left panel in Fig. 9). Compared to the ISM CR models, $N_{\mathrm{N}_{2} \mathrm{H}^{+}}$is reduced by a factor of a few around the peak and by more than an order of magnitude at larger radii in the low CR models. As a consequence the profile is also steeper. Although higher X-rays (CL_XH model) and also SPs can to some extent compensate low CR ionization rates, the $N_{\mathrm{N}_{2} \mathrm{H}^{+}}$profile is still steeper and $N_{\mathrm{N}_{2} \mathrm{H}^{+}}$is lower by a factor of between approximately two and six than in the ISM CR models for $r \gtrsim 30 \mathrm{au}$.

From Fig. 10 we see that only in the CL_XN_SP model SPs dominate $N_{\mathrm{N}_{2} \mathrm{H}^{+}}$for $r \gtrsim 70 \mathrm{au}$. Actually the importance of SPs increases with $r$ in this model. In the CL_XN_SP model SPs are the dominant ionization source in regions with $N_{\langle\mathrm{H}\rangle \text {,rad }} \lesssim 10^{25} \mathrm{~cm}^{-2}$. The $N_{\langle\mathrm{H}\rangle \text {,rad }}=10^{25} \mathrm{~cm}^{-2}$ iso-contour is below the vertical CO ice line for $r>70$ au and the layer between the CO ice line and $N_{\langle\mathrm{H}\rangle \text {,rad }}=10^{25} \mathrm{~cm}^{-2}$ becomes thicker with radius (see Figs. 7 and 8 ). This also explains the change in the slope of $\mathrm{N}_{\mathrm{N}_{2} \mathrm{H}^{+}}$compared to models without SPs.

In the high X-ray model, CL_XH_SP, the picture is quite different. $N_{\mathrm{N}_{2} \mathrm{H}^{+}}$is now dominated by X-rays for $r \gtrsim 30 \mathrm{au}$. $\mathrm{X}$-rays are efficiently scattered towards the midplane and therefore $\zeta_{\mathrm{X}}>\zeta_{\mathrm{SP}}$ in the cold $\mathrm{N}_{2} \mathrm{H}^{+}$layer (see also Fig. 7). X-rays affect the cold $\mathrm{N}_{2} \mathrm{H}^{+}$layer at all radii therefore the slope of $\mathrm{N}_{\mathrm{N}_{2} \mathrm{H}^{+}}$ is steeper than in the model where SP dominate (compare the blue solid line with the red dashed line in Fig. 9).
Our results show that $\mathrm{HCO}^{+}$is always significantly affected by SP ionization but $\mathrm{N}_{2} \mathrm{H}^{+}$only in models with low CRs and normal X-rays (for $r>30 \mathrm{au}$ ). As SPs can only reach the upper layers of the cold molecular layer, $\mathrm{N}_{2} \mathrm{H}^{+}$is less sensitive to SP ionization than $\mathrm{HCO}^{+}$.

\subsubsection{Impact of SPs in the Turner model}

In the Turner model SP ionization is just a scaled up version of CR ionization where SPs can also penetrate the disk vertically (see Appendix D). The results of the Turner models are also shown in Figs. 9 and 10.

In the ISM CR models there is no significant impact on $\mathrm{HCO}^{+}$and $\mathrm{N}_{2} \mathrm{H}^{+}$by SP ionization. $\zeta_{\mathrm{SP}}$ is significantly lower at low column densities compared to our models (see Sect. 3.1). Therefore X-rays are the dominant ionization source in the warm molecular layer and $\mathrm{HCO}^{+}$is not significantly affected by SP ionization. The slight increase in the $\mathrm{HCO}^{+}$column density is mainly due to the higher X-ray luminosity in the Turner model compared to our reference model with normal X-rays. Similar to our models CRs dominate in the cold molecular layer.

In the Turner model with low CRs, SPs become the dominant ionization source in the cold molecular layer as they also penetrate the disk vertically. In this layer $\zeta_{\text {SP }}$ reaches values of $\approx 10^{-17} \mathrm{~s}^{-1}$ at $r \approx 100 \mathrm{au}$. However, in the Turner model SPs also cannot compensate for a low CR ionization rate as $\zeta_{\mathrm{SP}} \propto 1 / r^{2}$ (geometric dilution).

The impact of SPs in the Turner model is rather limited and restricted to the cold molecular layer, in strong contrast to our models. The differences are mainly due to the assumptions concerning the SP transport. In the Turner model SPs hit the surface of the disk and penetrate the disk vertically, whereas we assume that SPs travel only along radial rays. However, both approaches are an approximation of a likely more complex picture of SP transport in disks. We discuss this in more detail in Sect. 4.3.

\section{Discussion}

\subsection{Constraining the SP flux of T Tauri stars}

SP ionization has a significant impact on the column densities of $\mathrm{HCO}^{+}$and $\mathrm{N}_{2} \mathrm{H}^{+}$in all our models. To actually constrain the SP flux from observations it is necessary to disentangle the contribution of SP ionization from the competing ionization sources CRs and X-rays.

From a chemical point of view all three ionization sources act the same way, they ionize molecular hydrogen and drive the molecular ion chemistry. However, they also show distinct differences in how they irradiate and penetrate the disk. CRs act like a background source and irradiate the disk isotropically, whereas X-rays and SPs originate from the star and act like a point source. A further difference is their energy distribution. Because of their high energies, CRs and SPs tend to move on straight lines whereas (hard) X-rays also experience scattering during their interaction with the disk.

Those differences in their irradiation properties and their energy distribution allow to disentangle their impact on the ion chemistry at different locations in the disk. Our models show that the stellar ionization sources are more effective closer to the star and at the surface layers of the disk (Fig. 10). X-rays can also become an important ionization source in the midplane of the disk but not for the whole disk as, roughly speaking, the ionization rate of stellar ionization sources is $\propto 1 / r^{2}$. CR ionization affects the whole disk, but is, in contrast to the stellar ionization 

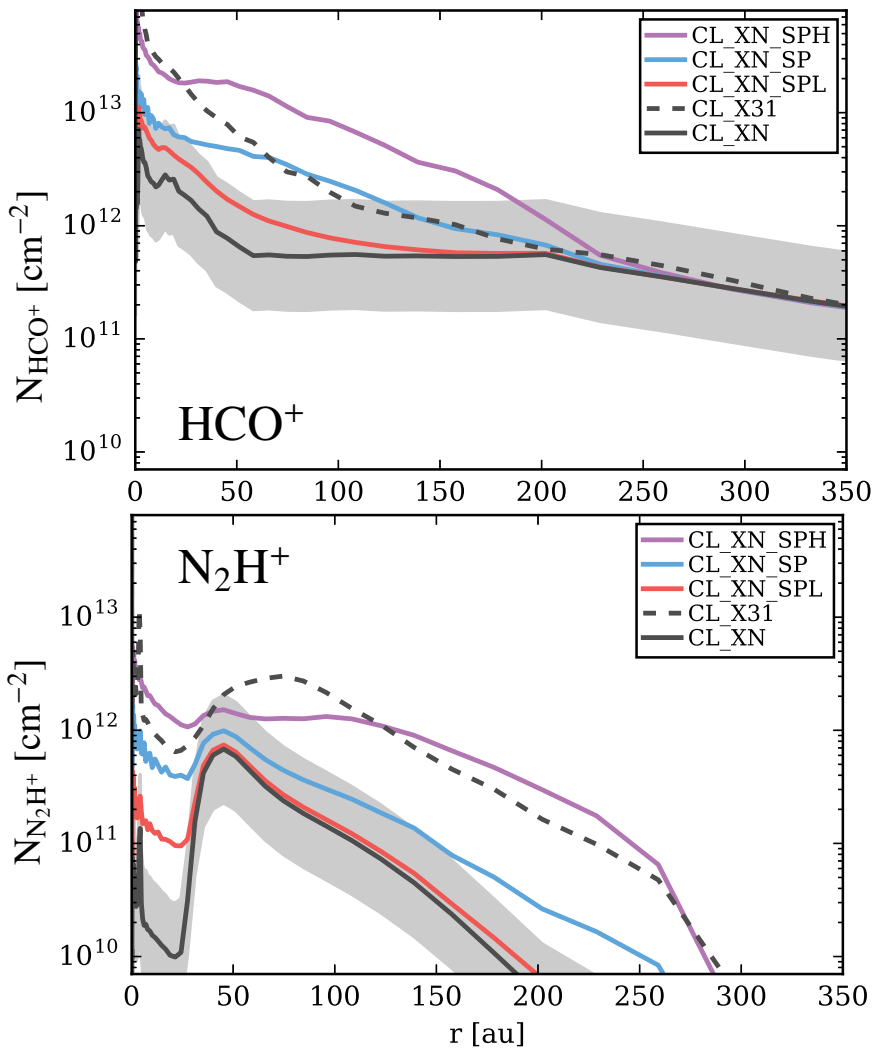

Fig. 11. Comparison of column densities for $\mathrm{HCO}^{+}$(top) and $\mathrm{N}_{2} \mathrm{H}^{+}$ (bottom) for models with varying SP flux and very strong X-ray emission. For all models the low CR ionization rates are used. Shown are the reference model with normal X-rays (black, CL_XN), the model with the typical SP flux (blue, CL_XN_SP) and models with a factor of ten higher (purple, $\mathrm{CL}_{-} \mathrm{XN} \_\mathrm{SPH}$ ) and factor of ten lower (red, CL_XN_SPL) SP flux. The dashed black line shows the model with $L_{\mathrm{X}}=3 \times 10^{31} \mathrm{erg} \mathrm{s}^{-1}$ and no SPs. The grey shaded area marks a difference of a factor of three in the column densities relative to the reference model CL_XN.

sources, more important for the outer disk and the cold layers and the midplane of the disk (this argument also holds for SLR ionization).

So far we have only shown models using the commonly proposed SP flux of $f_{\mathrm{p}}\left(E_{\mathrm{p}} \geq 10 \mathrm{MeV}\right) \approx 10^{7}$ protons $\mathrm{cm}^{-2} \mathrm{~s}^{-1}$ (e.g. Feigelson et al. 2002). However, this value should be only seen as an order of magnitude estimate (see Sect. 2.2).

In Fig. 11 we show the column densities for $\mathrm{HCO}^{+}$and $\mathrm{N}_{2} \mathrm{H}^{+}$for models with a SP flux a factor of ten higher and lower with respect to the reference value. Also shown are the reference model for the low $\mathrm{CR}$ case $\mathrm{CL}_{-} \mathrm{XN}$ and the model with the reference SP flux CL_XN_SP. For these models we use the low $\mathrm{CR}$ ionization rate $\left(\bar{\zeta}_{\mathrm{CR}} \approx 2 \times 10^{-19} \mathrm{~s}^{-1}\right)$ and the normal X-ray luminosity $\left(L_{\mathrm{X}}=10^{30} \mathrm{erg} \mathrm{s}^{-1}\right)$. Further we show a model with $L_{\mathrm{X}}=3 \times 10^{31} \mathrm{erg} \mathrm{s}^{-1}$, to illustrated the impact of (very) strong $\mathrm{X}$-ray emission (e.g. $\zeta_{\mathrm{X}} \gtrsim 10^{-17} \mathrm{~s}^{-1}$ in the disk midplane).

In the low SP flux model (CL_XN_SPL) the impact on $\mathrm{HCO}^{+}$and $\mathrm{N}_{2} \mathrm{H}^{+}$is quite limited. For such a case it is still possible to define upper limits for the SP flux in disks.

In the high SP flux model (CL_XN_SPH) the SP ionization rate reaches values of $\zeta_{\mathrm{SP}} \gtrsim 10^{-13} \mathrm{~s}^{-1}$ and $\zeta_{\mathrm{SP}} \approx 10^{-17} \mathrm{~s}^{-1}$ in the warm and cold molecular layer respectively. The $\mathrm{N}_{2} \mathrm{H}^{+}$column density profile beyond the radial $\mathrm{CO}$ ice line is comparable to the profile for models with ISM CRs (compare with Fig. 9). Also the model with very high X-rays (CL_X31) and no SPs shows a similar profile for $\mathrm{N}_{2} \mathrm{H}^{+}$. However, as seen from Fig. 11 the corresponding $\mathrm{HCO}^{+}$profiles differ significantly.

Comparing the very high X-ray model (CL_X31) to the reference model with SPs (CL_XN_SP) shows that the corresponding $\mathrm{HCO}^{+}$profiles are similar but the $\mathrm{N}_{2} \mathrm{H}^{+}$profiles differ by an order of magnitude. This shows again that it is indeed possible to distinguish between the different ionization sources by simultaneous modelling of $\mathrm{HCO}^{+}$and $\mathrm{N}_{2} \mathrm{H}^{+}$column density profiles.

To trace this interplay of ionization sources, spatially resolved observations of molecular ion lines tracing different vertical layers of the disk are required. With modern (sub)millimetre interferometers like the Atacama Large Millimeter Array (ALMA), the NOrthern Extended Millimeter Array (NOEMA) and the Submillimeter Array (SMA) such observations with a spatial resolution of tens of au are already possible (e.g. Qi et al. 2013b; Cleeves et al. 2015; ALMA Partnership et al. 2015; Yen et al. 2016) and will become available on a regular basis in the near future. Here, we use $\mathrm{HCO}^{+}$and $\mathrm{N}_{2} \mathrm{H}^{+}$as the tracers of the warm and cold molecular layer respectively, but also other molecules like $\mathrm{DCO}^{+}$, which traces similar regions as $\mathrm{N}_{2} \mathrm{H}^{+}$ (Teague et al. 2015; Mathews et al. 2013) can be used.

Complementary, far-infrared lines of $\mathrm{HCO}^{+}$and $\mathrm{N}_{2} \mathrm{H}^{+}$, as used by Ceccarelli et al. (2014) to trace SP ionization in a protostellar envelope, are good tracers of molecular ion emission in the warm inner region of the disk. However, a more detailed analysis with proper modelling of line emission is required to identify the best observational tracers of SP ionization. We will present such an analysis in a follow-up paper.

\subsection{Chemical implications}

Besides the $\mathrm{H}_{2}$ ionization rates there are other "chemical parameters", which have an impact on the molecular ion abundance in disks. In the following we discuss the dependence of our results on the location of the $\mathrm{CO}$ ice line, depletion of $\mathrm{CO}$ and the assumed initial metal abundances. Those chemical properties of disks are not well constrained from observations and/or can vary between different targets.

\subsubsection{Location of the $\mathrm{CO}$ ice line}

Recent ALMA observations provide direct constrains on the location of the $\mathrm{CO}$ ice line. However, these results depend on the method or more precisely the molecule used to trace the $\mathrm{CO}$ ice line (see Qi et al. 2013b; Schwarz et al. 2016; Nomura et al. 2016, for TW Hya). Further, due to complex chemical processes like the $\mathrm{CO}$ sink effect it is possible that the actual location of the $\mathrm{CO}$ ice line does not only depend on the $\mathrm{CO}$ freeze-out temperature (Aikawa et al. 2015, Sect. 3.3.2).

To investigate the dependence of our results on the location of the CO ice line we artificially moved the CO ice line in our model by adapting the binding energy for $\mathrm{CO}$. We consider two cases: $E_{\mathrm{B}}(\mathrm{CO})=950 \mathrm{~K}$ and $E_{\mathrm{B}}(\mathrm{CO})=1350 \mathrm{~K}$ (i.e $200 \mathrm{~K}$ lower and higher compared to our reference model). In both cases we kept the ratio of $E_{\mathrm{B}}\left(N_{2}\right) / E_{\mathrm{B}}(\mathrm{CO})=0.67$ constant (see Appendix B.2.2). As a consequence also the $\mathrm{N}_{2} \mathrm{H}^{+}$ layer moves accordingly to the $\mathrm{CO}$ ice line (see Sect. 3.3.1). For $E_{\mathrm{B}}(\mathrm{CO})=950 \mathrm{~K}$ the $\mathrm{CO}$ ice line moves to $T_{\mathrm{d}} \approx 20-24 \mathrm{~K}$, (i.e. deeper into the disk) and for $E_{\mathrm{B}}(\mathrm{CO})=1350 \mathrm{~K}$ to $T_{\mathrm{d}} \approx 25-36 \mathrm{~K}$ (i.e. higher up in the disk).

For a $\mathrm{CO}$ ice line deeper in the disk the contribution of $\mathrm{CR}$ ionization to the total column of $\mathrm{HCO}^{+}$and $\mathrm{N}_{2} \mathrm{H}^{+}$increases in the ISM CR models. In the ISM CR models SP ionization is not 
significant anymore (i.e. $N_{\mathrm{HCO}^{+}}$increases by less than a factor two). However, in the low CR models the impact of SPs remains significant.

A CO ice line higher up in the disk has the opposite effect. The total column densities of the molecular ions are now dominated by layers higher up in the disk which can efficiently be ionized by SPs. As a consequence the relative contribution of SP ionization to $\mathrm{N}_{\mathrm{HCO}^{+}}$and $\mathrm{N}_{\mathrm{N}_{2} \mathrm{H}^{+}}$increases.

In summary for a $\mathrm{CO}$ ice line location deeper in the disk SP ionization becomes less important, for a $\mathrm{CO}$ ice line higher up in the disk SP ionization becomes more important. However, in both cases the interplay of the different ionization sources is qualitatively speaking similar to what is shown in Fig. 10.

\subsubsection{CO depletion}

There is observational evidence for CO depletion in protoplanetary disks (Dutrey et al. 1997; Bruderer et al. 2012; Favre et al. 2013; Kama et al. 2016a; Schwarz et al. 2016; McClure et al. 2016). The best constraint case is TW Hya. Using spatially resolved ALMA spectral line observations of several CO isotopologues Schwarz et al. (2016) derived a uniform CO abundance of $\approx 10^{-6}$ in the warm molecular layer, two order of magnitudes lower than the canonical value of $\approx 10^{-4}$. However, the degree of $\mathrm{CO}$ depletion seems to vary from source to source. Using Herschel HD $J=1-0$ line observations McClure et al. (2016) derived $\mathrm{CO}$ depletions of a factor of approximately five and up to $\approx 100$ for DM Tau and GM Aur, respectively.

The cause of CO depletion in disks is not yet clear. Although freeze-out of CO certainly contributes to depletion it is unlikely that it is the only process acting. Several other mechanisms that can at least partly explain $\mathrm{CO}$ depletion are proposed:

- the destruction of $\mathrm{CO}$ by $\mathrm{He}^{+}$and the subsequent conversion of atomic carbon to more complex carbon bearing molecules with higher freeze-out temperatures (Aikawa et al. 1996; Bergin et al. 2014; Helling et al. 2014; Furuya \& Aikawa 2014);

- depletion of $\mathrm{CO}$ in layers above the $\mathrm{CO}$ ice line (up to $T \approx$ $30 \mathrm{~K}$ ) due to conversion of $\mathrm{CO}$ to $\mathrm{CO}_{2}$ on the surfaces of dust grains (Reboussin et al. 2015);

- CO isotopologue selective photodissociation, which affects $\mathrm{CO}$ isotopologue line emission and therefore the derived $\mathrm{CO}$ depletion factors;

- carbon and/or oxygen depletion in the warm disk atmosphere due to settling and mixing of ice coated dust grains (Du et al. 2015; Kama et al. 2016b).

It is as yet unclear which of the proposed mechanisms is the most efficient one; none of them can be excluded with certainty. It is also possible that all of these processes are at work. So far the impact of $\mathrm{CO}$ (carbon and/or oxygen depletion) on molecular ion emission was not studied in detail. For the modelling of $\mathrm{HCO}^{+}$and $\mathrm{N}_{2} \mathrm{H}^{+}$line emission of TW Hya, Cleeves et al. (2015) reduced the initial atomic carbon abundance by two orders of magnitude to match $\mathrm{C}^{18} \mathrm{O}$ line observation. However, the impact of $\mathrm{C}$ and $\mathrm{CO}$ depletion on $\mathrm{HCO}^{+}$and $\mathrm{N}_{2} \mathrm{H}^{+}$was not discussed in detail.

To simulate CO depletion we simply reduce the total carbon and oxygen element abundances by one order of magnitude throughout the disk. This results in a $\mathrm{CO}$ abundance of $\approx 10^{-5}$ in the warm molecular layer. We applied this "artificial" CO depletion to all models listed in Table 5; all other parameters of the models are fixed.
The CO depletion models show a factor of $\approx 5-10$ lower $N_{\mathrm{HCO}^{+}}$for $r \gtrsim 50$ au compared to the non depleted models. For $r<30$ au $N_{\mathrm{N}_{2} \mathrm{H}^{+}}$increases by more than an order of magnitude. $N_{\mathrm{N}_{2} \mathrm{H}^{+}}$beyond the CO ice line is not affected as $N_{\mathrm{N}_{2} \mathrm{H}^{+}}$resides within the $\mathrm{CO}$ freeze-out zone where gas phase $\mathrm{CO}$ is anyway depleted.

In the $\mathrm{CO}$ depletion models SP ionization is slightly more efficient for $N_{\mathrm{N}_{2} \mathrm{H}^{+}}$as the contribution of the warm $\mathrm{N}_{2} \mathrm{H}^{+}$layer to $\mathrm{N}_{\mathrm{N}_{2} \mathrm{H}^{+}}$increases. Due to the lower $\mathrm{CO}$ abundance in the warm molecular layer the $\mathrm{N}_{2} \mathrm{H}^{+}$abundance increases as the destruction pathway via $\mathrm{CO}$ is less efficient. For $\mathrm{HCO}^{+}$the opposite is true. The $\mathrm{HCO}^{+}$abundance decreases by roughly an order of magnitude in the warm molecular layer, whereas in the $\mathrm{CO}$ freezeout zone the impact is smaller (i.e. $\mathrm{CO}$ is frozen-out anyway). Relatively speaking the contribution of the cold $\mathrm{HCO}^{+}$to $\mathrm{N}_{\mathrm{HCO}^{+}}$ increases in the $\mathrm{CO}$ depletion models. Therefore the impact of SPs on $N_{\mathrm{HCO}^{+}}$is less significant whereas X-rays and CRs become more important. Although there are some differences, the impact of SPs on $N_{\mathrm{N}_{2} \mathrm{H}^{+}}$and $N_{\mathrm{HCO}^{+}}$is qualitatively very similar to the non-depleted models. In particular the main trends derived from Fig. 10 are also seen in the $\mathrm{CO}$ depletion models.

$\mathrm{CO}$ depletion is certainly more complex than modelled here. A more thorough study of the impact of CO depletion on molecular ion abundances is certainly desirable and possibly provides new constraints on $\mathrm{CO}$ gas phase depletion in disks. However, this is beyond the scope of this paper.

\subsubsection{Metal abundances}

Heavy metals such as sulphur play an important role in the molecular ion disk chemistry (Teague et al. 2015, Rab et al., in prep.). Here we refer with the term metals to the elements $\mathrm{Na}, \mathrm{Mg}, \mathrm{Si}, \mathrm{S}$ and $\mathrm{Fe}$. Metal ionization due to UV radiation can produce a large number of free electrons. Those free electrons destroy molecular ions like $\mathrm{HCO}^{+}$and $\mathrm{N}_{2} \mathrm{H}^{+}$via dissociative recombination. Dissociative recombination is more efficient than radiative recombination of metals. As a consequence a high abundance of metals significantly reduces the abundance of molecular ions (e.g. Mitchell et al. 1978; Graedel et al. 1982).

In the ISM and in disks most of the metals are likely locked up in refractory grains and are therefore depleted compared to Solar abundances. We use metal abundances similar to the commonly used "low metal" abundances (Graedel et al. 1982; Lee et al. 1998a). These low metal abundances are depleted by a factor of $\approx 100-1000$ compared to Solar abundances (i.e. low metal sulphur abundance $\epsilon(S) \approx 10^{-7}$ ). However, the actual gas phase abundance of metals is difficult to constrain from observations and a stronger degree of depletion already prior to disk formation is possible (e.g. Maret \& Bergin 2007; Maret et al. 2013).

To investigate the dependence of our results on the metal abundances we deplete the initial gas phase metal abundance by an additional factor of ten compared to the low metal abundances (i.e. $\epsilon(\mathrm{S}) \approx 10^{-8}$ ). This means that a larger fraction of metals is locked-up in refractory dust grains and cannot be released back into the gas phase.

Decreasing the metal abundances increases $\epsilon\left(\mathrm{HCO}^{+}\right)$in the warm molecular layer by up to an order of magnitude (i.e. the gap in the vertical $\epsilon\left(\mathrm{HCO}^{+}\right)$profile nearly vanishes; Sect. 3.3.1). In the cold molecular layer the metal abundances are not as important since the metals are frozen-out on dust grains anyway. However, in regions where non-thermal desorption processes are efficient (i.e. metal ices are released back into the gas-phase), $\epsilon\left(\mathrm{HCO}^{+}\right)$but also $\epsilon\left(\mathrm{N}_{2} \mathrm{H}^{+}\right)$are higher by a factor of a few in the lower metal abundance models. The $\mathrm{HCO}^{+}$column density 
is higher by a factor of between approximately two and three at all radii in the strong metal depletion models compared to the models with the reference abundances. The $\mathrm{N}_{2} \mathrm{H}^{+}$column density is only affected for $r \gtrsim 150$ au (higher by a factor of between approximately two and three), where non-thermal desorption of metals is efficient.

Lower gas phase metal abundances lead to an increase of the molecular ion abundances in the warm molecular layer and the contribution of this layer to the total column densities increases. However, in the strong metal depletion models the interplay and the relative contributions to the column densities of the different ionization sources is nearly identical to the reference model grid (i.e. Fig. 10 does not change significantly). Our arguments concerning the impact of SPs on $\mathrm{HCO}^{+}$and $\mathrm{N}_{2} \mathrm{H}^{+}$are therefore also valid for the case of strong metal depletion.

\subsection{Future prospects}

Our model results show that SPs can indeed become an important ionization source in $\mathrm{T}$ Tauri disks. However, our model should be seen as a first attempt at a comprehensive modelling of SP ionization in protoplanetary disks. In the following we discuss further important aspects like variability, non-stellar origin of SPs, the importance of magnetic fields and future prospects for SP modelling in protoplanetary disks.

\subsubsection{Flares and variability}

In our models we assumed continuous (i.e. time-averaged) particle fluxes and X-ray luminosities. Although this is a reasonable assumption (see Sect. 2.2) it is likely that the disk is also hit by singular powerful X-ray and/or SP flares.

From the X-ray COUP survey of the ONC cloud, Feigelson et al. (2002) and Wolk et al. (2005) derived a median X-ray flare luminosity of $\approx 6 \times 10^{30} \mathrm{erg} \mathrm{s}^{-1}$, comparable to the X-ray luminosity in our high X-ray models, and peak flare luminosities up to $10^{31} \mathrm{erg} \mathrm{s}^{-1}$. The duration of such flares can last from hours up to three days with a typical frequency of roughly one powerful flare per week.

Ilgner \& Nelson (2006) argue that for such flare properties the disk ion chemistry responds to time-averaged ionization rates. However, in case the duration between two flares is longer than the recombination time scale in the disk, such singular and strong flares would produce asymmetric features in molecular ion emission (i.e. a singular flare only affects a certain fraction of the disk). The spatially resolved $\mathrm{HCO}^{+} J=3-2$ SMA observations of TW Hya show indeed such an asymmetric structure (Cleeves et al. 2015). However, as discussed by Cleeves et al. (2015) these features could also have a different origin like spiral arms or an hidden planet locally heating the disk.

If such asymmetric structures are caused by stellar flares they would provide complementary constraints on the X-ray/SP activity of the star. Multi-epoch data of spatially resolved molecular ion emission is required to prove the flare scenario (i.e. the features should disappear quickly). The current (sub)mm interferometers like ALMA, NOEMA and SMA provide the required spatial resolution and might even allow for monitoring of disks in molecular ion lines on a daily/weekly basis in the future.

Modelling of such observations does not necessarily require 3D chemical models. Assuming that the disk physical structure is not affected by flares, radial cuts through the disk can be modelled with 2D (time-dependent) thermo-chemical models as presented here.

\subsubsection{Magnetic fields}

In our model we neglect the impact of magnetic fields on the SP transport. Stellar and disk magnetic fields are in particular relevant for the question if particles actually hit the disk (see Feigelson et al. 2002, for a discussion).

Magnetic fields can either drag the particles away from the disk (e.g. like in a wind) but could also funnel the particles and concentrate the ionizing flux in particular regions of the disk. In the first scenario SPs can still have an impact on the upper layers of the disk but certainly not on the disk midplane. In the second scenario particles are likely focused on regions close to the inner radius of the disk and their impact on the outer disk will become less significant. Also the trajectory of the particles will be affected, and they might penetrate the disk also vertically. Our models, where particles are transported only radially, are closer to the wind scenario whereas the Turner model would represent an extreme case for magnetically focused particles. However, to qualitatively estimate the impact of magnetic fields more complex SP transport models are required.

It is possible to consider magnetic field effects in high energy particle transport models (Desch et al. 2004; Padovani \& Galli 2011; Padovani et al. 2013b). In principle such methods can also be applied to disk models. The main challenge though is to determine the structure of the star and disk magnetic fields. However, as argued by Ceccarelli et al. (2014) identifying distinct observational signatures of SP ionization in disks and/or envelopes would allow to derive constrains for the magnetic field structure. This is certainly challenging, but with the availability of spatially resolved observational data and interpretation of such data with (improved) thermo-chemical disk models this might be feasible in the near future.

\subsubsection{Non-stellar origin of high energy particles}

Besides the stellar surface the close environment of young stars offers also alternative particle acceleration sites. X-ray flares and particles can be produced close to the inner disk in the so called reconnection ring where the stellar and disk magnetic field interact (X-wind model Shu et al. 1994, 1997). More recently Padovani et al. $(2015,2016)$ proposed jet shocks as alternative acceleration sites for high energy particles.

In the $\mathrm{X}$-wind model the particle source and also the X-ray emitting source is located closer to the disk and slightly above the disk midplane. The typically assumed source location in this "lamppost" scenario is $r_{\mathrm{L}} \approx 0.05 \mathrm{au}$ and $z_{\mathrm{L}} \approx 0.05 \mathrm{au}\left(\approx 10 R_{\odot}\right)$ (Lee et al. 1998b; Igea \& Glassgold 1999). For X-rays Ercolano et al. (2009) found that the height of the emitting source has relatively little impact concerning X-ray radiative transfer in disks. Moving the emitting source closer to the disk certainly has an impact on the very inner disk but for e.g. 10 au distance from the star the stellar particle flux increases only by about $1 \%$ compared to the stellar origin. The height of the SP emitting source has some impact on where in the disk SPs see a column density $N_{\langle\mathrm{H}\rangle} \gtrsim 10^{25} \mathrm{~cm}^{-2}$. For particles accelerated at a height $z_{\mathrm{L}} \approx 0.05 \mathrm{au}$ and moving along a ray parallel to the midplane this happens at $r \approx 0.5 \mathrm{au}$. The consequences are that SPs can penetrate into slightly deeper layers of the disks but they still cannot penetrate to the disk midplane. Therefore our conclusions on the impact of SPs on $\mathrm{HCO}^{+}$and $\mathrm{N}_{2} \mathrm{H}^{+}$remain valid for the $\mathrm{X}$-wind scenario.

In the jet shock scenario, the emitting source would be located far above the disk. Padovani et al. (2016) considered a particle emitting source located at $1.8 \times 10^{3}$ au above the star and 
calculated the resulting ionization rates for a 2D disk structure. Depending on their particle acceleration model they found ionization rates up to $\zeta \approx 10^{-14} \mathrm{~s}^{-1}$ at the surface layers of the disk. Although X-ray ionization rates in the upper layer of the disk are typically higher such an irradiation scenario could have a significant impact on the ionization of the outer disk $(r \gtrsim 50 \mathrm{au})$. In the outer disk, the jet accelerated particles can penetrate the disk vertically and therefore can also reach the disk midplane (similar to the Turner model, Sect. 3.3.4). This might become important if Galactic CRs are efficiently attenuated (i.e. in the low CR case).

The scenarios described above for non-stellar particle sources are certainly worth being investigated in detail. For the future we plan to extend our model to a proper treatment of such non-stellar emitting sources.

\section{Summary and conclusions}

In this work we investigated the impact of stellar energetic particle (SP) ionization on disk chemistry with a focus on the common disk ionization tracers $\mathrm{HCO}^{+}$and $\mathrm{N}_{2} \mathrm{H}^{+}$. We assumed a typical SP flux of $f_{\mathrm{p}}\left(E_{\mathrm{p}} \approx 10 \mathrm{MeV}\right) \approx 10^{7}$ protons $\mathrm{cm}^{-2} \mathrm{~s}^{-1}$ (at $1 \mathrm{au}$ ) as commonly proposed for $\mathrm{T}$ Tauri stars and a particle energy distribution derived from measurements of solar particle events. Based on a detailed particle transport model we derived an easy to use formula (see Sect. 2.2) to calculate the SP ionization rate in the disk as a function of hydrogen column density and radius, assuming that the particles can penetrate the disk only radially. With a small grid of models considering varying properties of the competing high energy disk ionization sources, X-rays and Galactic cosmic rays, we studied the interplay of the different ionization sources and identified possible observational tracers of SP ionization. Our main conclusions are the following:

- SPs cannot penetrate the disk midplane. At hydrogen column densities $N_{\langle\mathrm{H}\rangle} \gtrsim 10^{25} \mathrm{~cm}^{-2}$ even the most energetic particles are attenuated (stopped) and the SP ionization rate drops rapidly. As the radial hydrogen column densities for full T Tauri disks are typically $N_{\langle\mathrm{H}\rangle} \gg 10^{25} \mathrm{~cm}^{-2}$ the midplane SP ionization rate is $\zeta_{\mathrm{SP}} \ll 10^{-20} \mathrm{~s}^{-1}$ already at a distance of 1 au from the star.

- For the assumed SP flux (see above), SPs become the dominant $\mathrm{H}_{2}$ ionization source in the warm molecular layer of the disk above the $\mathrm{CO}$ ice line, provided that SPs are not shielded by magnetic fields. This is even true for enhanced X-ray luminosities (i.e. $L_{\mathrm{X}}=5 \times 10^{30} \mathrm{erg} \mathrm{s}^{-1}$ ).

- SP ionization can increase the $\mathrm{HCO}^{+}$and $\mathrm{N}_{2} \mathrm{H}^{+}$column densities by factors of between approximately three and ten for disk radii $r \lesssim 200 \mathrm{au}$. The impact is more significant in models with low CR ionization rates (i.e. $\zeta_{\mathrm{CR}} \approx 10^{-19} \mathrm{~s}^{-1}$ ).

- SP ionization becomes insignificant for an SP flux one order of magnitude lower than the proposed value for $\mathrm{T}$ Tauri stars. In such a case $\mathrm{H}_{2}$ ionization is solely dominated by X-rays and CRs.

- As SPs cannot penetrate the deep layers of the disk, X-rays and/or CRs usually remain the dominant $\mathrm{H}_{2}$ ionization source in the cold disk layers (i.e. below the $\mathrm{CO}$ ice line). Therefore $\mathrm{HCO}^{+}$, which traces the warm molecular layer, is more sensitive to SP ionization than $\mathrm{N}_{2} \mathrm{H}^{+}$that resides in the cold molecular layer.

- Simultaneous modelling of spatially resolved radial intensity profiles of molecular ions tracing different vertical layers of the disk allows to disentangle the contributions of the competing high energy ionization sources to the total $\mathrm{H}_{2}$ ionization rate. Consequently such observations allow to constrain the SP flux in disks. Such a method is likely to be model dependent and ancillary observations constraining the vertical chemical structure of disks are required.

We have shown that stellar energetic particles can be an important ionization agent for disk chemistry. Modelling of spatially resolved observations of molecular ions with a model such as presented here allows to put first constraints on the stellar particle flux in disks around T Tauri stars.

Further model improvements concerning the stellar energetic particle transport (i.e. magnetic fields) are required to answer the question to what extent stellar particles reach the disk. Additionally non-stellar origins (i.e. jets) of high energy particles should be considered. With such models and spatially resolved molecular ion observations it will be possible to put stringent constraints on stellar energetic particle fluxes of $\mathrm{T}$ Tauri stars and to infer properties of the stellar and disk magnetic fields.

Acknowledgements. The authors thank the anonymous referee for useful suggestions and comments. We want to thank C. Dominik for very helpful discussions on stellar particle transport in disks. The research leading to these results has received funding from the European Union Seventh Framework Programme FP7-2011 under grant agreement No. 284405. R.CH. acknowledges funding by the Austrian Science Fund (FWF): project number P24790. M.P. acknowledges funding from the European Unions Horizon 2020 research and innovation programme under the Marie Skłodowska-Curie grant agreement No. 664931. The computational results presented have been achieved using the Vienna Scientific Cluster (VSC). This publication was supported by the Austrian Science Fund (FWF). This research has made use of NASA's Astrophysics Data System. All figures were made with the free Python module Matplotlib (Hunter 2007).

\section{References}

Adams, N., Smith, D., \& Grief, D. 1978, International Journal of Mass Spectrometry and Ion Physics, 26, 405

Aikawa, Y., Miyama, S. M., Nakano, T., \& Umebayashi, T. 1996, ApJ, 467, 684 Aikawa, Y., Umebayashi, T., Nakano, T., \& Miyama, S. M. 1997, ApJ, 486, L51 Aikawa, Y., Furuya, K., Nomura, H., \& Qi, C. 2015, ApJ, 807, 120

ALMA Partnership, Brogan, C. L., Pérez, L. M., et al. 2015, ApJ, 808, L3

Andrews, S. M., Wilner, D. J., Hughes, A. M., Qi, C., \& Dullemond, C. P. 2009, ApJ, 700, 1502

Aresu, G., Kamp, I., Meijerink, R., et al. 2011, A\&A, 526, A163

Bergeron, H., Rougeau, N., Sidis, V., et al. 2008, J. Phys. Chem. A, 112, 11921 Bergin, E. A., \& Langer, W. D. 1997, ApJ, 486, 316

Bergin, E. A., Ciardi, D. R., Lada, C. J., Alves, J., \& Lada, E. A. 2001, ApJ, 557, 209

Bergin, E. A., Cleeves, L. I., Crockett, N., \& Blake, G. A. 2014, Faraday Discussions, 168, 61

Bethell, T. J., \& Bergin, E. A. 2011, ApJ, 740, 7

Bisschop, S. E., Fraser, H. J., Öberg, K. I., van Dishoeck, E. F., \& Schlemmer, S. 2006, A\&A, 449, 1297

Bohme, D. K., Mackay, G. I., \& Schiff, H. I. 1980, J. Chem. Phys., 73, 4976

Borget, F., Chiavassa, T., Allouche, A., \& Aycard, J. P. 2001, J. Phys. Chem. B, 105, 449

Botschwina, P., Horn, M., Flugge, J., \& Seeger, S. 1993, J. Chem. Soc., Faraday Trans., 89, 2219

Brott, I., \& Hauschildt, P. H. 2005, in The Three-Dimensional Universe with Gaia, eds. C. Turon, K. S. O’Flaherty, \& M. A. C. Perryman, ESA SP, 576, 565

Bruderer, S., van Dishoeck, E. F., Doty, S. D., \& Herczeg, G. J. 2012, A\&A, 541, A91

Ceccarelli, C., \& Dominik, C. 2005, A\&A, 440, 583

Ceccarelli, C., Dominik, C., López-Sepulcre, A., et al. 2014, ApJ, 790, L1

Cleeves, L. I., Adams, F. C., \& Bergin, E. A. 2013, ApJ, 772, 5

Cleeves, L. I., Bergin, E. A., \& Adams, F. C. 2014, ApJ, 794, 123

Cleeves, L. I., Bergin, E. A., Qi, C., Adams, F. C., \& Öberg, K. I. 2015, ApJ, 799, 204

Cleeves, L. I., Bergin, E. A., O’D. Alexander, C. M., et al. 2016, ApJ, 819, 13 Collings, M. P., Anderson, M. A., Chen, R., et al. 2004, MNRAS, 354, 1133

Desch, S. J., Connolly, Jr., H. C., \& Srinivasan, G. 2004, ApJ, 602, 528

Draine, B. T., \& Bertoldi, F. 1996, ApJ, 468, 269

Du, F., Bergin, E. A., \& Hogerheijde, M. R. 2015, ApJ, 807, L32

Dubrulle, B., Morfill, G., \& Sterzik, M. 1995, Icarus, 114, 237 
Dulieu, F., Congiu, E., Noble, J., et al. 2013, Sci. Rep., 3, 1338 Dullemond, C. P., \& Dominik, C. 2004, A\&A, 421, 1075

Dutrey, A., Guilloteau, S., \& Guelin, M. 1997, A\&A, 317, L55

Dutrey, A., Henning, T., Guilloteau, S., et al. 2007, A\&A, 464, 615

Dutrey, A., Semenov, D., Chapillon, E., et al. 2014, Protostars and Planets VI, 317

Ercolano, B., \& Glassgold, A. E. 2013, MNRAS, 436, 3446

Ercolano, B., Clarke, C. J., \& Drake, J. J. 2009, ApJ, 699, 1639

Favre, C., Cleeves, L. I., Bergin, E. A., Qi, C., \& Blake, G. A. 2013, ApJ, 776 L38

Fayolle, E. C., Balfe, J., Loomis, R., et al. 2016, ApJ, 816, L28

Feigelson, E. D., \& Montmerle, T. 1999, ARA\&A, 37, 363

Feigelson, E. D., Garmire, G. P., \& Pravdo, S. H. 2002, ApJ, 572, 335

Flower, D. R. 1999, MNRAS, 305, 651

France, K., Schindhelm, E., Bergin, E. A., Roueff, E., \& Abgrall, H. 2014, ApJ, 784, 127

Furuya, K., \& Aikawa, Y. 2014, ApJ, 790, 97

Garrod, R. T., \& Herbst, E. 2006, A\&A, 457, 927

Glassgold, A. E., Najita, J., \& Igea, J. 1997, ApJ, 480, 344

Glassgold, A. E., Feigelson, E. D., Montmerle, T., \& Wolk, S. 2005, in Chondrites and the Protoplanetary Disk, eds. A. N. Krot, E. R. D. Scott, \& B. Reipurth, ASP Conf. Ser., 341, 165

Glassgold, A. E., Galli, D., \& Padovani, M. 2012, ApJ, 756, 157

Glauser, A. M., Güdel, M., Watson, D. M., et al. 2009, A\&A, 508, 247

Gounelle, M., Shu, F. H., Shang, H., et al. 2001, ApJ, 548, 1051

Gounelle, M., Shu, F. H., Shang, H., et al. 2006, ApJ, 640, 1163

Graedel, T. E., Langer, W. D., \& Frerking, M. A. 1982, ApJS, 48, 321

Güdel, M., \& Nazé, Y. 2009, A\&ARv, 17, 309

Güdel, M., Briggs, K. R., Arzner, K., et al. 2007, A\&A, 468, 353

Güdel, M., Lahuis, F., Briggs, K. R., et al. 2010, A\&A, 519, A113

He, J., Jing, D., \& Vidali, G. 2014, Phys. Chem. Chem. Phys. (Incorporating Faraday Transactions), 16, 3493

Helling, C., Woitke, P., Rimmer, P. B., et al. 2014, Life, 4, 142

Herbst, E., Payzant, J. D., Schiff, H. I., \& Bohme, D. K. 1975, ApJ, 201, 603

Hily-Blant, P., Walmsley, M., Pineau Des Forêts, G., \& Flower, D. 2010, A\&A, 513, A41

Hunter, J. D. 2007, Comput. Sci. Eng., 9, 90

Igea, J., \& Glassgold, A. E. 1999, ApJ, 518, 848

Ilgner, M., \& Nelson, R. P. 2006, A\&A, 455, 731

Kama, M., Bruderer, S., Carney, M., et al. 2016a, A\&A, 588, A108

Kama, M., Bruderer, S., van Dishoeck, E. F., et al. 2016b, A\&A, 592, A83

Kamp, I., Tilling, I., Woitke, P., Thi, W.-F., \& Hogerheijde, M. 2010, A\&A, 510, A18

Kamp, I., Thi, W.-F., Woitke, P., et al. 2017, A\&A, submitted

Klippenstein, S. J., Georgievskii, Y., \& McCall, B. J. 2010, J. Phys. Chem. A, 114,278

Lawson, P. A., Osborne, D., Jr., \& Adams, N. G. 2011, Int. J. Mass Spectr., 304, 41

Le Gal, R., Hily-Blant, P., Faure, A., et al. 2014, A\&A, 562, A83

Lee, H.-H., Roueff, E., Pineau des Forets, G., et al. 1998a, A\&A, 334, 1047

Lee, T., Shu, F. H., Shang, H., Glassgold, A. E., \& Rehm, K. E. 1998b, ApJ, 506, 898

Li, X., Heays, A. N., Visser, R., et al. 2013, A\&A, 555, A14

Lynden-Bell, D., \& Pringle, J. E. 1974, MNRAS, 168, 603

Maret, S., \& Bergin, E. A. 2007, ApJ, 664, 956

Maret, S., Bergin, E. A., \& Tafalla, M. 2013, A\&A, 559, A53

Mathews, G. S., Klaassen, P. D., Juhász, A., et al. 2013, A\&A, 557, A132

McClure, M. K., Bergin, E. A., Cleeves, L. I., et al. 2016, ApJ, 831, 167

McElroy, D., Walsh, C., Markwick, A. J., et al. 2013, A\&A, 550, A36

McKeegan, K. D., Chaussidon, M., \& Robert, F. 2000, Science, 289, 1334

Meijerink, R., Aresu, G., Kamp, I., et al. 2012, A\&A, 547, A68

Mewaldt, R. A., Cohen, C. M. S., Labrador, A. W., et al. 2005, J. Geophys. Res. (Space Phys.), 110, 9

Mewaldt, R. A., Cohen, C. M. S., Mason, G. M., Haggerty, D. K., \& Desai, M. I. 2007, Space Sci. Rev., 130, 323
Meyer, B. S., \& Clayton, D. D. 2000, Space Sci. Rev., 92, 133

Min, M., Hovenier, J. W., \& de Koter, A. 2005, A\&A, 432, 909

Min, M., Rab, C., Woitke, P., Dominik, C., \& Ménard, F. 2016, A\&A, 585, A13

Minissale, M., Congiu, E., \& Dulieu, F. 2014, J. Chem. Phys., 140, 074705

Minissale, M., Congiu, E., \& Dulieu, F. 2016a, A\&A, 585, A146

Minissale, M., Dulieu, F., Cazaux, S., \& Hocuk, S. 2016b, A\&A, 585, A24

Mitchell, J. B. A. 1990, Phys. Rep., 186, 215

Mitchell, G. F., Kuntz, P. J., \& Ginsburg, J. L. 1978, ApJS, 38, 39

Noble, J. A., Congiu, E., Dulieu, F., \& Fraser, H. J. 2012, MNRAS, 421, 768

Nomura, H., Tsukagoshi, T., Kawabe, R., et al. 2016, ApJ, 819, L7

Öberg, K. I., van Broekhuizen, F., Fraser, H. J., et al. 2005, ApJ, 621, L33

Öberg, K. I., Qi, C., Fogel, J. K. J., et al. 2010, ApJ, 720, 480

Öberg, K. I., Qi, C., Fogel, J. K. J., et al. 2011a, ApJ, 734, 98

Öberg, K. I., Qi, C., Wilner, D. J., \& Andrews, S. M. 2011b, ApJ, 743, 152

Padovani, M., \& Galli, D. 2011, A\&A, 530, A109

Padovani, M., Galli, D., \& Glassgold, A. E. 2009, A\&A, 501, 619

Padovani, M., Galli, D., \& Glassgold, A. E. 2013a, A\&A, 549, C3

Padovani, M., Hennebelle, P., \& Galli, D. 2013b, A\&A, 560, A114

Padovani, M., Hennebelle, P., Marcowith, A., \& Ferrière, K. 2015, A\&A, 582, L13

Padovani, M., Marcowith, A., Hennebelle, P., \& Ferrière, K. 2016, A\&A, 590, A8

Payzant, J. D. 1975, J. Chem. Phys., 63, 149

Qi, C., Kessler, J. E., Koerner, D. W., Sargent, A. I., \& Blake, G. A. 2003, ApJ, 597,986

Qi, C., Öberg, K. I., \& Wilner, D. J. 2013a, ApJ, 765, 34

Qi, C., Öberg, K. I., Wilner, D. J., et al. 2013b, Science, 341, 630

Qi, C., Öberg, K. I., Andrews, S. M., et al. 2015, ApJ, 813, 128

Rakshit, A. 1982, International Journal of Mass Spectrometry and Ion Physics, 41, 185

Reames, D. V. 2013, Space Sci. Rev., 175, 53

Reames, D. V. 2015, Space Sci. Rev., 194, 303

Reboussin, L., Wakelam, V., Guilloteau, S., Hersant, F., \& Dutrey, A. 2015, A\&A, 579, A82

Reedy, R. C. 2012, Lun. Planet. Sci. Conf., 43, 1285

Schöier, F. L., van der Tak, F. F. S., van Dishoeck, E. F., \& Black, J. H. 2005, A\&A, 432, 369

Schoonjans, T., Brunetti, A., Golosio, B., et al. 2011, Spectrochim. Acta, 66, 776 Schwarz, K. R., Bergin, E. A., Cleeves, L. I., et al. 2016, ApJ, 823, 91

Shu, F., Najita, J., Ostriker, E., et al. 1994, ApJ, 429, 781

Shu, F. H., Shang, H., Glassgold, A. E., \& Lee, T. 1997, Science, 277, 1475

Takayanagi, K. 1973, PASJ, 25, 327

Teague, R., Semenov, D., Guilloteau, S., et al. 2015, A\&A, 574, A137

Thi, W.-F., Woitke, P., \& Kamp, I. 2011, MNRAS, 412, 711

Thi, W.-F., Pinte, C., Pantin, E., et al. 2014, A\&A, 561, A50

Tielens, A. G. G. M., \& Allamandola, L. J. 1987, in Interstellar Processes, eds. D. J. Hollenbach, \& H. A. Thronson, Jr., ASSL, 134, 397

Trappitsch, R., \& Ciesla, F. J. 2015, ApJ, 805, 5

Turner, N. J., \& Drake, J. F. 2009, ApJ, 703, 2152

Umebayashi, T., \& Nakano, T. 2009, ApJ, 690, 69

Wakelam, V., Herbst, E., Loison, J.-C., et al. 2012, ApJS, 199, 21

Wakelam, V., Loison, J.-C., Herbst, E., et al. 2015, ApJS, 217, 20

Wakelam, V., Smith, I. W. M., Loison, J.-C., et al. 2013, ArXiv e-prints [arXiv: 1310.4350$]$

Ward, M. D., Hogg, I. A., \& Price, S. D. 2012, MNRAS, 425, 1264

Webber, W. R. 1998, ApJ, 506, 329

Williams, J. P., \& Cieza, L. A. 2011, ARA\&A, 49, 67

Woitke, P., Kamp, I., \& Thi, W.-F. 2009, A\&A, 501, 383

Woitke, P., Riaz, B., Duchêne, G., et al. 2011, A\&A, 534, A44

Woitke, P., Min, M., Pinte, C., et al. 2016, A\&A, 586, A103

Wolk, S. J., Harnden, Jr., F. R., Flaccomio, E., et al. 2005, ApJS, 160, 423

Yen, H.-W., Liu, H. B., Gu, P.-G., et al. 2016, ApJ, 820, L25 


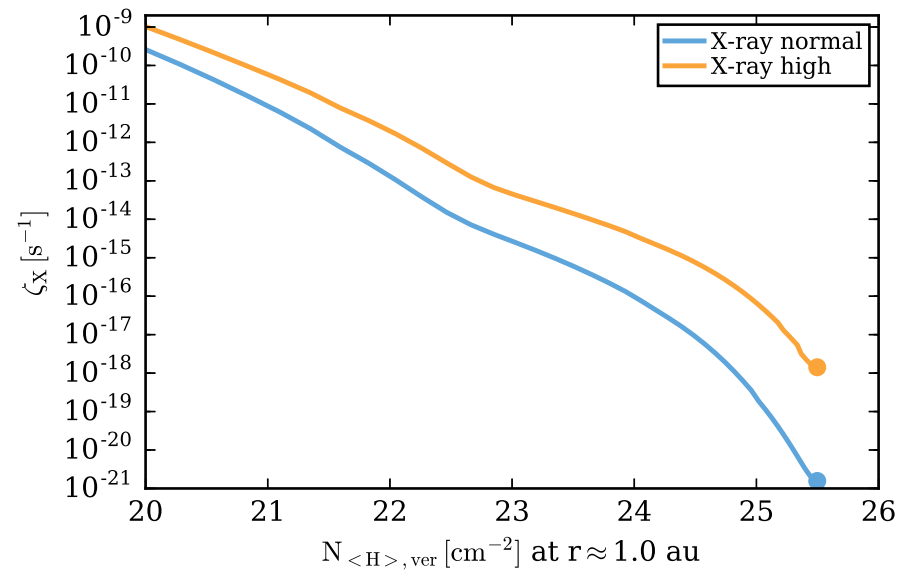

Fig. A.1. X-ray ionization rate $\zeta_{\mathrm{X}}$ as a function of vertical hydrogen column density of the disk at a distance of 1 au from the star. Shown are the resulting ionization rates for the two different X-ray spectra (see Sect. 2.3.1). The dots mark the same location $(r=1 \mathrm{au}, z=0 \mathrm{au})$ in the disk as the dots in Fig. A.2.

\section{Appendix A: X-ray radiative transfer}

Here we have used the newly developed X-ray radiative transfer module of PRODIMO. The details of this module will be presented in a forthcoming paper Rab et al. (in prep.). Here we only briefly describe the implementation and present results concerning the X-ray ionization rate for comparison with other models.

The main difference between the new model and the existing implementation of Aresu et al. (2011), Meijerink et al. (2012) is the treatment of X-ray scattering. We used the absorption and scattering cross-section from the publicly available xraylib library ${ }^{1}$ (Schoonjans et al. 2011). For the X-ray radiative transfer we use the same numerical approach (e.g. use of wavelength bands) as is used for the dust radiative transfer in ProDiMo (Woitke et al. 2009). We assumed isotropic scattering but applied a correction factor (anisotropic factor $\mathrm{g}$ ) to account for anisotropic Compton scattering. According to Cleeves et al. (2016), neglecting anisotropic scattering has only a limited impact on the X-ray ionization rate of about a factor of two. We consider this possible deviation as not significant for the results presented here. We further disregarded the dust in the X-ray radiative transfer, since with the assumed gas to dust ratio of 100 and dust settling X-ray photons mainly interact with the gas (Bethell \& Bergin 2011; Glassgold et al. 2012).

The X-ray chemistry in ProDiMo is presented in detail in Meijerink et al. (2012). The interaction of X-rays with the gas changes the species abundances and consequently also the $\mathrm{X}$-ray opacities. We therefore iterated between the X-ray radiative transfer and the chemistry until convergence is reached.

In Figs. A. 1 and A. 2 we show the X-ray ionization rate $\zeta_{\mathrm{X}}$ for our disk model (Sect. 2.4) and for both X-ray spectra (Sect. 2.3.1). Figure A.1 shows $\zeta_{\mathrm{X}}$ as a function of vertical column density at 1 au whereas Fig. A. 2 shows $\zeta_{X}$ in the midplane of the disk as a function of radius. These results are in good quantitative agreement with Ercolano \& Glassgold (2013) and Cleeves et al. (2015) considering that different disk models and different implementations of X-ray radiative transfer (e.g. crosssections) are applied.

\footnotetext{
1 https://github.com/tschoonj/xraylib
}

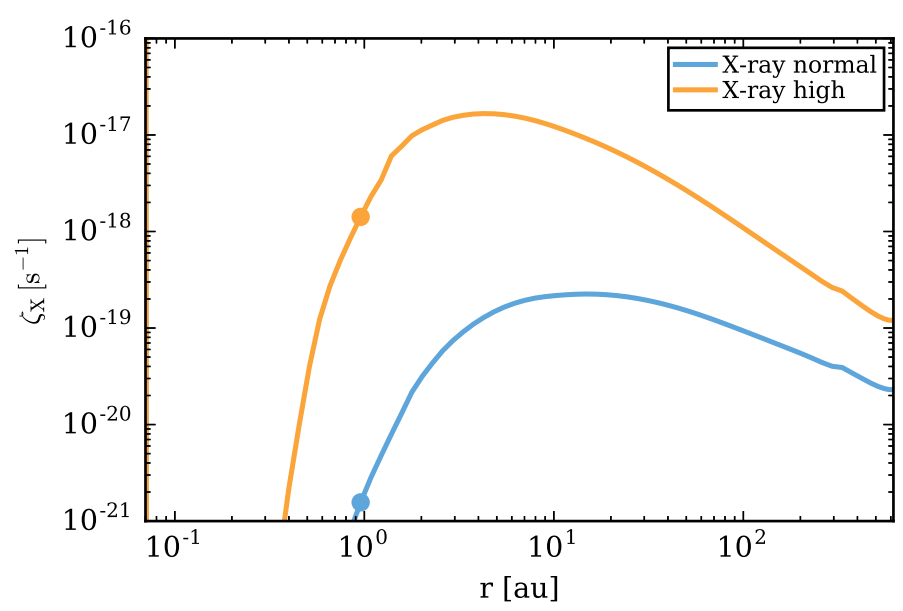

Fig. A.2. X-ray ionization rate $\zeta_{\mathrm{x}}$ as a function of radius in the midplane of the disk $(z=0)$. Shown are the resulting ionization rates for the two different X-ray spectra (see Sect. 2.3.1). The dots mark the same location $(r=1 \mathrm{au}, z=0 \mathrm{au})$ in the disk as the dots in Fig. A.1.

\section{Appendix B: Chemistry}

For the chemical reaction network we use the UMIST 2012 database for gas phase chemistry (McElroy et al. 2013) for a selection of 235 species. Additionally the network includes X-ray chemistry (Aresu et al. 2011; Meijerink et al. 2012), PAH (polycyclic aromatic hydrocarbons) chemistry (Thi et al. 2014), adsorption and desorption (thermal, CR and photo desorption) for ice species and $\mathrm{H}_{2}$ formation on grains (Woitke et al. 2009). In total the network includes 3143 chemical reactions. For the ice species we use the binding energies from the UMIST 2012 release but updated a couple of values for oxygen bearing species (see Sect. B.2). Further details on the network can be found in Kamp et al. (2017). The most relevant gas phase reactions for $\mathrm{HCO}^{+}$and $\mathrm{N}_{2} \mathrm{H}^{+}$with their rate coefficients are listed in Table B.1.

To test the robustness of our chemical model with respect to $\mathrm{HCO}^{+}$and $\mathrm{N}_{2} \mathrm{H}^{+}$we performed chemical tests with time-dependent chemistry, varying binding energies and the KIDA chemical network (Kinetic Database for Astrochemistry, Wakelam et al. 2012, 2015). The different test models are described in Table B.2 and discussed in the following sections. The results for the $\mathrm{HCO}^{+}$and $\mathrm{N}_{2} \mathrm{H}^{+}$column densities are shown in Figs. B. 1 and B.2.

\section{B.1. $\mathrm{N}_{2}$ shielding}

Dust shielding of $\mathrm{N}_{2}$ photodissociation is strongly reduced in protoplanetary disks due to dust evolution (e.g. dust growth) and shielding by $\mathrm{H}_{2}$ becomes important (Li et al. 2013). To account for this we implemented the $\mathrm{H}, \mathrm{H}_{2}$ shielding and self-shielding functions for $\mathrm{N}_{2}$ of Li et al. (2013) in our chemistry model. The data are taken from the Leiden photodissociation database $\mathrm{e}^{2}$.

We find that $\mathrm{N}_{2}$ shielding is very important for the abundance structure of $\mathrm{N}_{2} \mathrm{H}^{+}$. In models without $\mathrm{N}_{2}$ shielding the vertical column density of $\mathrm{N}_{2} \mathrm{H}^{+}$is reduced by one order of magnitude throughout the disk. Further the warm $\mathrm{N}_{2} \mathrm{H}^{+}$layer above the vertical CO ice-line vanishes.

\footnotetext{
2 http://home.strw.leidenuniv.nl/ heays/photo/
} 
Table B.1. Rate coefficients for the main formation and destruction pathways of $\mathrm{HCO}^{+}$and $\mathrm{N}_{2} \mathrm{H}^{+}$.

\begin{tabular}{lcccccc}
\hline \hline Reaction & $\alpha$ & $\beta$ & $\gamma$ & $\begin{array}{c}a \\
k(25 \mathrm{~K}) \\
\left(\mathrm{cm}^{3} \mathrm{~s}^{-1}\right)\end{array}$ & Type & Reference \\
\hline $\mathrm{H}_{3}^{+}+\mathrm{CO} \rightarrow \mathrm{H}_{2}+\mathrm{HCO}^{+}$ & $1.35(-9)$ & -0.14 & -3.4 & $2.2(-9)$ & $\begin{array}{c}\text { Ion-neutral } \\
\text { DCO }\end{array}$ \\
$\mathrm{HCO}^{+}+\mathrm{e}_{2} \mathrm{O} \rightarrow \mathrm{HO}+\mathrm{H}_{3} \mathrm{O}^{+}$ & $2.40(-7)$ & -0.69 & 0.0 & $1.3(-6)$ & Dissociative recombination & 2 \\
$\mathrm{HCO}_{3}^{+}+\mathrm{N}_{2} \rightarrow \mathrm{H}_{2}+\mathrm{N}_{2} \mathrm{H}^{+}$ & $1.80(-9)$ & -0.50 & 0.0 & $8.7(-9)$ & Ion-neutral & 3 \\
$\mathrm{~N}_{2} \mathrm{H}^{+}+\mathrm{e}^{-} \rightarrow \mathrm{H}+\mathrm{N}_{2}$ & $2.77(-7)$ & -0.87 & 0.0 & $1.8(-9)$ & Ion-neutral & 4 \\
$\mathrm{~N}_{2} \mathrm{H}^{+}+\mathrm{e}^{-} \rightarrow \mathrm{NH}+\mathrm{N}$ & $2.09(-8)$ & -0.74 & 0.0 & $1.3(-7)$ & Dissociative recombination & 5 \\
$\mathrm{~N}_{2} \mathrm{H}^{+}+\mathrm{CO} \rightarrow \mathrm{N}_{2}+\mathrm{HCO}^{+}$ & $8.8(-10)$ & 0.0 & 0.0 & $8.8(-10)$ & Ion-neutral & 6 \\
\hline
\end{tabular}

Notes. The rate coefficient is given by the modified Arrhenius equation $k(T)=\alpha \times(T / 300 \mathrm{~K}) \times \exp (-\gamma / T)$.

References. UMIST 2012 database (McElroy et al. 2013); (1) Klippenstein et al. (2010); (2) Mitchell (1990); (3) Adams et al. (1978); (4) Rakshit (1982); (5) Lawson et al. (2011); (6) Payzant (1975), Herbst et al. (1975), Bohme et al. (1980).

Table B.2. Chemical test models.

\begin{tabular}{ll}
\hline \hline Name & Description \\
\hline EBUMIST & $\begin{array}{l}\text { original binding energies from } \\
\text { UMIST 2012 }\end{array}$ \\
EBN2 & $\begin{array}{l}\mathrm{N}_{2} \text { to CO binding energy ratio } \\
\text { of 0.9 according to experiments } \\
\text { no } \mathrm{N}_{2} \text { shielding } \\
\text { N2SH } \\
\text { KIDA2011 phase chemical network from } \\
\text { the KIDA 2011 release } \\
\text { KIDA2014 }\end{array}$ \\
& $\begin{array}{l}\text { gas phase chemical network from } \\
\text { the KIDA 2014 release }\end{array}$ \\
\hline
\end{tabular}

Notes. All chemical test models are based on the reference model CI_XN.

\section{B.2. Binding energies}

\section{B.2.1. Oxygen binding energy}

Several laboratory experiments (e.g. Ward et al. 2012; He et al. 2014; Minissale et al. 2016a,b) reported binding energies for oxygen in the range of $E_{\mathrm{B}}(\mathrm{O})=1500-1800 \mathrm{~K}$. This is significantly higher than the value of $E_{\mathrm{B}}(\mathrm{O})=800 \mathrm{~K}$ listed in the UMIST 2012 database. We therefore updated the binding energies for oxygen and several other oxygen bearing species with the values listed in Minissale et al. (2016b). The new values with their references are given in Table B.3.

The higher binding energy for oxygen has a significant impact on $\mathrm{N}_{2} \mathrm{H}^{+}$. Using the UMIST 2012 binding energy reduces the $\mathrm{N}_{2} \mathrm{H}^{+}$column density by about a factor of three for $r \gtrsim 50 \mathrm{au}$ (see model EBUMIST in Fig. B.1). Similar to CO, oxygen also destroys $\mathrm{N}_{2} \mathrm{H}^{+}$via the reaction $\mathrm{O}+\mathrm{N}_{2} \mathrm{H}^{+} \rightarrow \mathrm{N}_{2}+\mathrm{OH}^{+}$. Due to this reaction the $\mathrm{N}_{2} \mathrm{H}^{+}$abundance is strongly reduced in a thin layer below the vertical $\mathrm{CO}$ ice line and above the vertical oxygen ice line. With the higher binding energy for oxygen this thin layer vanishes as oxygen freezes-out at higher temperatures than CO.

For $\mathrm{HCO}^{+}$the dip in the column density profile at $r \approx 50$ au alone is significantly affected. In this region $\mathrm{HCO}^{+}$is efficiently destroyed by water. Removing oxygen and $\mathrm{OH}$ from the gas phase reduces also the water abundance near the vertical $\mathrm{CO}$ ice line, consequently the $\mathrm{HCO}^{+}$abundance increases.
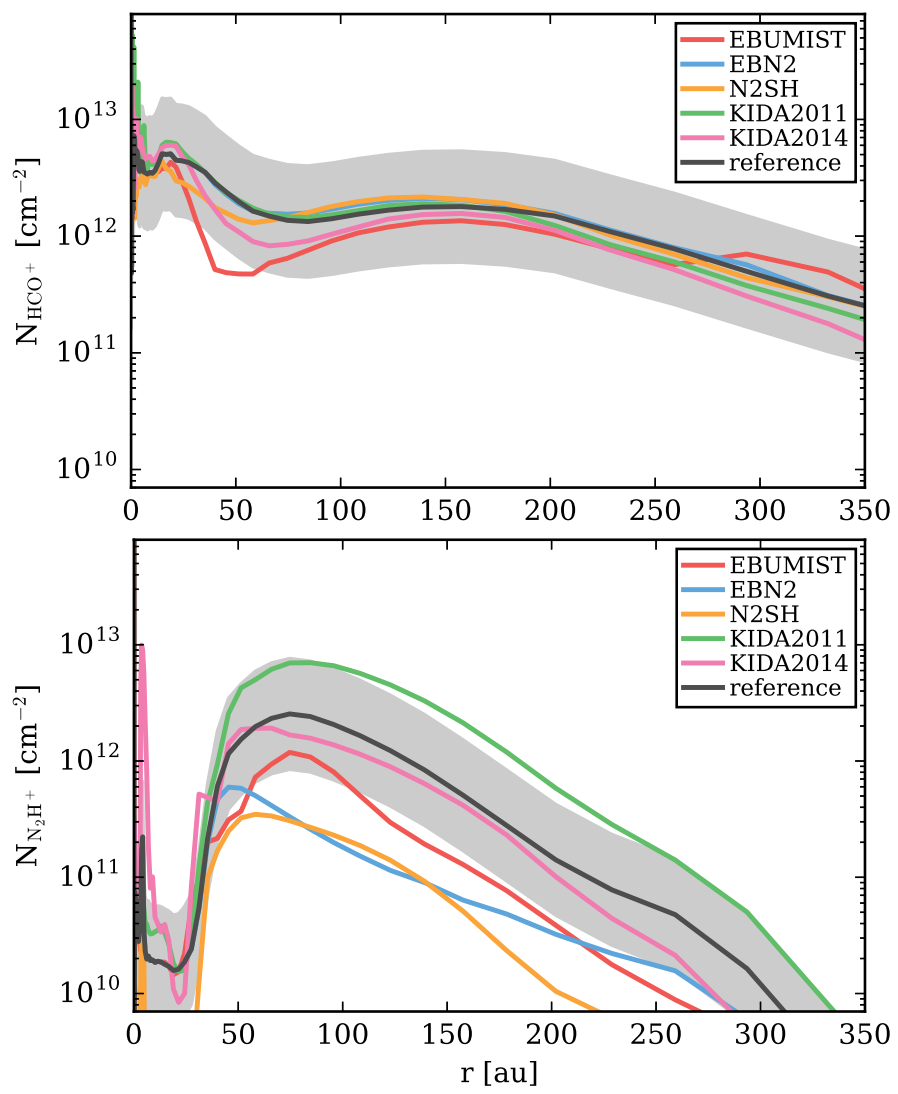

Fig. B.1. Comparison of column densities for $\mathrm{HCO}^{+}$and $\mathrm{N}_{2} \mathrm{H}^{+}$for models with different chemical networks or binding energies (see Table B.2). The grey shaded area marks a difference of a factor 3 in the column densities relative to the reference model (black line).

\section{B.2.2. $\mathrm{N}_{2}$ binding energy}

In the UMIST 2012 database $E_{\mathrm{B}}\left(\mathrm{N}_{2}\right)=0.67 \times E_{\mathrm{B}}(\mathrm{CO})$ (see Table B.3). This ratio is close to the value of 0.65 used to explain observations of prestellar cores showing that $\mathrm{N}_{2}$ freezes out at lower temperatures than CO (e.g. Bergin \& Langer 1997; Bergin et al. 2001; Ceccarelli \& Dominik 2005). Also disk observations of $\mathrm{N}_{2} \mathrm{H}^{+}$indicate that $E_{\mathrm{B}}\left(\mathrm{N}_{2}\right)<E_{\mathrm{B}}(\mathrm{CO})$ (Qi et al. 2003, $2013 \mathrm{~b}$ ). However, the ratio of 0.65 is in contradiction with laboratory experiments which show $E_{\mathrm{B}}\left(\mathrm{N}_{2}\right) \approx 0.9 \times E_{\mathrm{B}}(\mathrm{CO})$ (Öberg et al. 2005; Bisschop et al. 2006; Fayolle et al. 2016). One explanation for this discrepancy might be differential freeze-out but it 

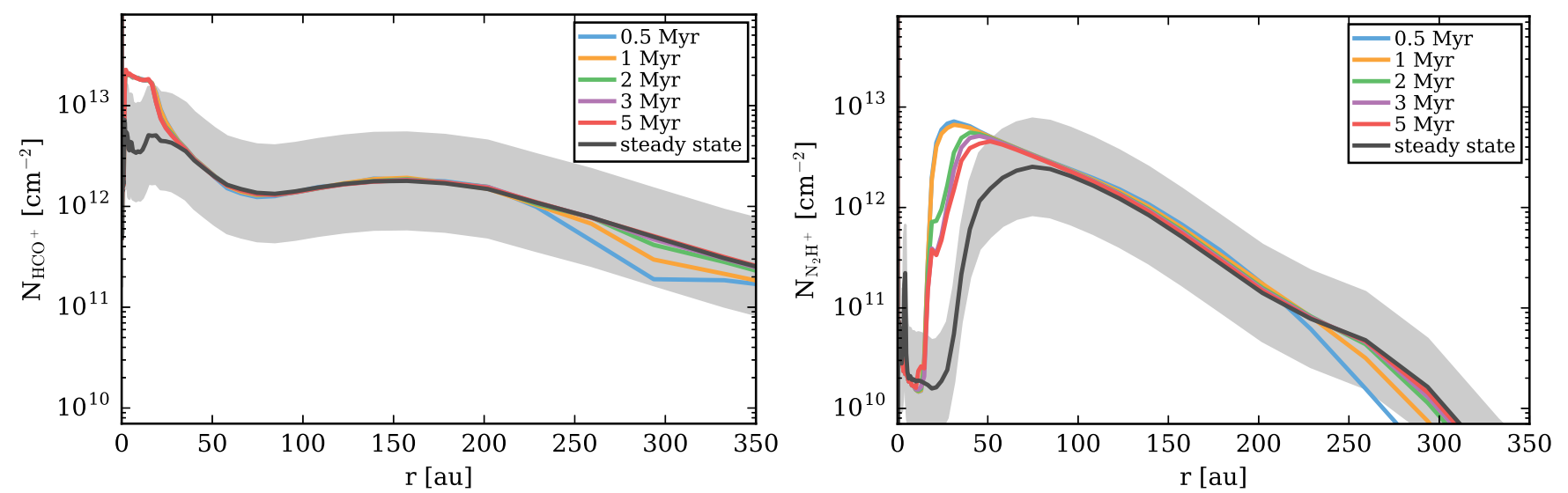

Fig. B.2. Comparison of time-dependent and steady-state chemistry models. Shown are the vertical column densities for $\mathrm{HCO}^{+}$and $\mathrm{N}_{2} \mathrm{H}^{+}$at different chemical ages of the disk (colored lines) and the steady-state results (black line). The grey shaded area marks a difference of a factor 3 in the column densities relative to the steady-state model.

Table B.3. Important binding energies.

\begin{tabular}{lcc}
\hline \hline Species & $\begin{array}{c}E_{\mathrm{B}} \text { UMIST 2012 } \\
(\mathrm{K})\end{array}$ & $\begin{array}{c}E_{\mathrm{B}} \\
(\mathrm{K})\end{array}$ \\
\hline $\mathrm{CO}$ & $1150^{a}$ & \\
$\mathrm{~N}_{2}$ & $790^{b}$ & \\
$\mathrm{O}$ & $800^{c}$ & $1500^{e}$ \\
$\mathrm{O}_{2}$ & $1000^{d}$ & $1250^{f}$ \\
$\mathrm{O}_{3}$ & $1800^{d}$ & $2100^{g}$ \\
$\mathrm{OH}$ & $2850^{d}$ & $4600^{h}$ \\
\hline
\end{tabular}

Notes. The second column shows the values from the UMIST 2012 database (McElroy et al. 2013), the third column the updated values from Minissale et al. (2016b).

References. ${ }^{(a)}$ Garrod \& Herbst (2006), Collings et al. (2004); ${ }^{(b)}$ Öberg et al. (2005); ${ }^{(c)}$ Tielens \& Allamandola (1987); ${ }^{(d)}$ Garrod \& Herbst (2006); ${ }^{(e)}$ Bergeron et al. (2008); ${ }^{(f)}$ Noble et al. (2012); ${ }^{(g)}$ Borget et al. (2001), Minissale et al. (2014); ${ }^{(h)}$ Dulieu et al. (2013).

is unclear if this is sufficient (Bisschop et al. 2006; Fayolle et al. 2016).

In our models the $\mathrm{N}_{2} \mathrm{H}^{+}$column density drops by about one order of magnitude if we set $E_{\mathrm{B}}\left(\mathrm{N}_{2}\right)=0.9 \times E_{\mathrm{B}}(\mathrm{CO})$ (model EBN2 in Fig. B.1). Contrary to our model Aikawa et al. (2015) find that in their disk model the $\mathrm{N}_{2} \mathrm{H}^{+}$abundance structure is not strongly affected by setting $E_{\mathrm{B}}\left(\mathrm{N}_{2}\right)=E_{\mathrm{B}}(\mathrm{CO})$. They argue that due to the sink effect, $\mathrm{CO}$ is depleted anyway and therefore the $\mathrm{N}_{2} \mathrm{H}^{+}$abundance is not strongly affected by setting $E_{\mathrm{B}}\left(\mathrm{N}_{2}\right)=E_{\mathrm{B}}(\mathrm{CO})$. We have not included dust surface chemistry in our model therefore the $\mathrm{CO}$ sink effect is less efficient. Aikawa et al. (2015) argue that the sink effect depends on various parameters (turbulence in the disk, efficiency of the conversion of $\mathrm{CO}$ to less volatile species) therefore they also studied a no-sink model. In this no-sink model the $\mathrm{N}_{2} \mathrm{H}^{+}$abundance is sensitive to the binding energy of $\mathrm{N}_{2}$, very similar to our models.

The main difference between the sink and no-sink model is the location of the $\mathrm{CO}$ ice line and the $\mathrm{N}_{2} \mathrm{H}^{+}$layer. In the sink model $\mathrm{CO}$ is also depleted at temperatures higher than its sublimation temperature, consequently the $\mathrm{CO}$ ice line moves to higher and warmer layers of the disk. In the no-sink model the $\mathrm{CO}$ ice line is given by the adsorption/desorption equilibrium for $\mathrm{CO}$ which depends on the chosen $\mathrm{CO}$ binding energy. However, with the exception of the exact location of the $\mathrm{CO}$ ice line and the $\mathrm{N}_{2} \mathrm{H}^{+}$layer our models with $E_{\mathrm{B}}\left(\mathrm{N}_{2}\right)=0.67 \times E_{\mathrm{B}}(\mathrm{CO})$ are in good agreement with the full chemical network/sink model of Aikawa et al. (2015). We discuss the impact of the CO ice line location on our results in Sect. 4.2.1.

\section{B.3. Comparison to the KIDA chemical network}

The reaction rates for nitrogen chemistry are not as well known as for carbon/oxygen chemistry (e.g. Hily-Blant et al. 2010; Le Gal et al. 2014). Wakelam et al. (2013) reviewed a large number of important reactions for nitrogen chemistry. The new derived reactions rates are in included in the latest KIDA gas phase chemistry database release (Wakelam et al. 2015).

We ran models using the KIDA gas phase chemistry database instead of the UMIST 2012 database (see Table B.2). We used the KIDA 2011 (Wakelam et al. 2012) and the KIDA 2014 (Wakelam et al. 2015) releases. The additional chemistry included in ProDiMo (e.g. X-ray chemistry) remains the same.

Figure B. 1 shows the resulting $\mathrm{HCO}^{+}$and $\mathrm{N}_{2} \mathrm{H}^{+}$column densities for the KIDA2011 and KIDA2014 model in comparison to the reference model (UMIST 2012). In the KIDA2011 model the $\mathrm{N}_{2} \mathrm{H}^{+}$column density is about a factor of three higher compared to the reference and KIDA2014 models. The reason are updated rate coefficients for the dissociative recombination reactions of $\mathrm{N}_{2} \mathrm{H}^{+}$with electrons. The reaction rates at $20 \mathrm{~K}$ for $\mathrm{N}_{2} \mathrm{H}^{+}+\mathrm{e}^{-} \rightarrow \mathrm{N}_{2}+\mathrm{H}\left(\mathrm{N}_{2} \mathrm{H}^{+}+\mathrm{e}^{-} \rightarrow \mathrm{NH}+\mathrm{N}\right)$ are a about a factor of five (three) higher in the KIDA 2014/UMIST 2012 releases than in the KIDA 2011 release. This explains the higher $\mathrm{N}_{2} \mathrm{H}^{+}$abundance in the KIDA 2011 model. However, we find a good agreement for the KIDA 2014 and the UMIST 2012 release for both molecules $\mathrm{HCO}^{+}$and $\mathrm{N}_{2} \mathrm{H}^{+}$.

\section{Appendix C: Steady-state versus time-dependent chemistry}

For our models we have assumed that the chemistry reaches a steady-state within typical lifetimes of disks (couple of million years). To verify this assumption we run time-dependent chemistry models for the reference models CI_XN and CL_XN. We find that for the species considered in this work $\mathrm{HCO}^{+}$and $\mathrm{N}_{2} \mathrm{H}^{+}$ the chemistry reaches steady-state within $\approx 1 \mathrm{Myr}$ in a large fraction of the disk. This is in agreement with the models of Aikawa et al. (2015) although they use a different, in particular larger chemical network.

In Fig. B. 2 we compare the vertical column densities $\mathrm{N}_{\mathrm{HCO}^{+}}$ and $N_{\mathrm{N}_{2} \mathrm{H}^{+}}$of the steady-state model to the results of the 
time-dependent models at different times. For $r \gtrsim 30$ au and $t \gtrsim 1$ Myr to column densities of the steady-state and the timedependent model are nearly identical. However, the steady-state model underestimates $N_{\mathrm{HCO}^{+}}$and $N_{\mathrm{N}_{2} \mathrm{H}^{+}}$for $r \lesssim 30$ au. The differences are caused by the "sink effect" for $\mathrm{CO}$ and $\mathrm{N}_{2}$ (see Sect. 3.3 and Aikawa et al. 2015). This erosion of $\mathrm{CO}$ and $\mathrm{N}_{2}$ and other neutral molecules in the disk midplane by the reaction with $\mathrm{He}^{+}$is a slow chemical process (e.g. Furuya \& Aikawa 2014; Bergin et al. 2014; Helling et al. 2014) and is therefore over-estimated in the steady-state models.

As $\mathrm{N}_{2} \mathrm{H}^{+}$resides in deeper layers than $\mathrm{HCO}^{+}$the deviations in the column densities are more pronounced. As a consequence the $\mathrm{N}_{2} \mathrm{H}^{+}$column density does not exactly trace the $\mathrm{CO}$ ice line in the midplane of the disk. The midplane $\mathrm{CO}$ ice line is located at $r \approx 12$ au in the time-dependent model, whereas in the steadystate model $N_{\mathrm{N}_{2} \mathrm{H}^{+}}$indicates a CO ice line at $r \approx 30 \mathrm{au}$. We note that in the low CR model CL_XN the sink effect is less efficient due to the lower midplane ionization rate, therefore the deviations in the steady-state models for $N_{\mathrm{HCO}^{+}}$and $N_{\mathrm{N}_{2} \mathrm{H}^{+}}$are smaller $\left(N_{\mathrm{N}_{2} \mathrm{H}^{+}}\right.$indicates a CO ice line at $\left.r \approx 20 \mathrm{au}\right)$.

Our comparison shows that for the bulk of $\mathrm{N}_{\mathrm{HCO}^{+}}$and $N_{\mathrm{N}_{2} \mathrm{H}^{+}}$ the assumption of steady-state chemistry is well justified. In particular our conclusions concerning the impact of SP ionization are not affected by the artifacts in the steady-state models. A steady-state model requires about a factor of ten less computational time than the time-dependent models. The use of the steady-state models allows us to study other important aspects like the impact of $\mathrm{CO}$ depletion (Sect. 4.2.2) or the comparison of different chemical networks.

\section{Appendix D: The Turner model}

In Turner \& Drake (2009) the SP ionization rate is calculated for a mean solar mass nebulae disk model. To calculate the SP ionization rate at the surface of the disk they scaled their CR ionization rate of $\zeta_{\mathrm{CR}}=5 \times 10^{-18} \mathrm{~s}^{-1}$ by a factor of $10^{4}(\mathrm{r} / \mathrm{au})^{-2}$. To account for the attenuation of SPs as a function of hydrogen column density they applied the same equation as they use for Galactic cosmic rays (their Eq. (2)).

To compare our results with the approach of Turner \& Drake (2009) we implemented their method in our model. We also used their X-ray input spectrum and applied our X-ray radiative transfer to calculate the X-ray ionization rate. In Fig. D. 1 we show the resulting X-ray, SP and CR ionization rates as a function of radius at a vertical column density of $\approx 8 \mathrm{~g} \mathrm{~cm}^{-2}\left(\approx 3 \times 10^{24} \mathrm{~cm}^{-2}\right)$ for the Turner model (CI_T) and for our CI_XN_SP model. A comparison of Fig. D.1 with Fig. 1 of Turner \& Drake (2009) shows that the CI_T model reproduces their SP ionization rates. We note that in our disk model (i.e lower disk mass) only for $r<10$ au a vertical column density of $>3 \times 10^{24} \mathrm{~cm}^{-2}$ is reached; therefore only the inner 10 au are shown.

Figure D.1 clearly shows the differences of the two approaches. In our models SPs can only penetrate the disk radially and therefore cannot reach the disk midplane (see Sect. 3.2). In the Turner model the particles can also penetrate the disk vertically down to the midplane as they use the same equation for SP attenuation as is used for Galactic CRs. We cannot reproduce their results if we allow only for radial transport of particles. The differences in the $\mathrm{X}$-ray ionization rate are due to the different X-ray spectrum $\left(L_{X}=2 \times 10^{30} \mathrm{erg} \mathrm{s}^{-1}, T_{X}=5.8 \times 10^{7} \mathrm{~K}\right.$, see Table 2) used by Turner \& Drake (2009).
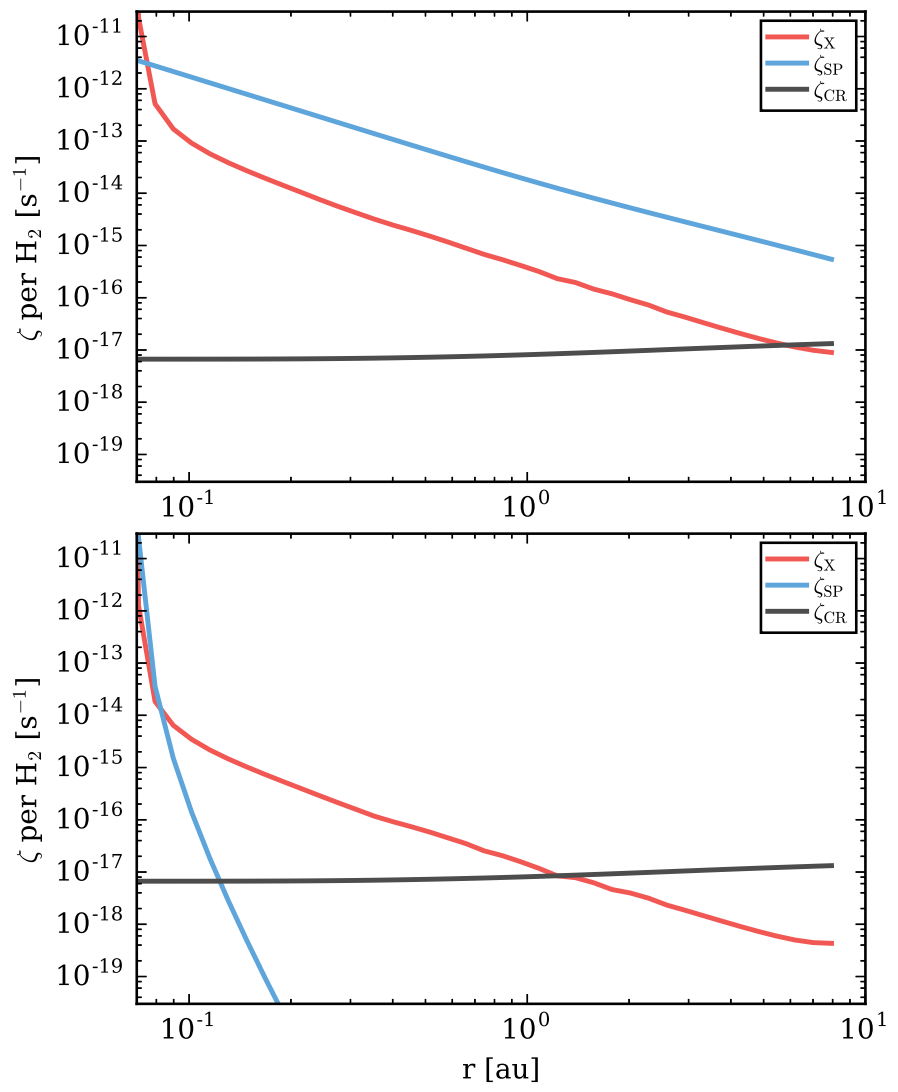

Fig. D.1. Ionization rates at a vertical column density of $N_{\langle\mathrm{H}\rangle \text {,ver }}=3.4 \times$ $10^{24} \mathrm{~cm}^{-2}\left(\approx 8 \mathrm{~g} \mathrm{~cm}^{-2}\right)$ as a function of distance to the star. The top panel shows the Turner model (CI_T), the bottom panel the CI_XN_SP model (see Table 5).

\section{Appendix E: Comparison to observations}

We compared the $\mathrm{HCO}^{+} J=3-2$ and $\mathrm{N}_{2} \mathrm{H}^{+} J=3-2$ line fluxes from our reference model to the observations of Öberg et al. (2010, 2011a). From their sample we choose the targets where both lines were detected (the six targets shown in Fig. 6 of Öberg et al. 2011a). The observed integrated line fluxes are in the range of $3.1-17.8 \mathrm{Jy} \mathrm{km} \mathrm{s}^{-1}$ for $\mathrm{HCO}^{+} J=3-2$ and in the range of $0.4-2.9 \mathrm{Jy} \mathrm{km} \mathrm{s}^{-1}$ for $\mathrm{N}_{2} \mathrm{H}^{+} J=3-2$ (all line fluxes are scaled to a distance of $140 \mathrm{pc}$ ). Excluding IM Lup, an extremely large and massive disk, from the sample gives a much narrower range of $3.1-5.4 \mathrm{Jy} \mathrm{km} \mathrm{s}^{-1}$ and $0.4-1.4 \mathrm{Jy} \mathrm{km} \mathrm{s}^{-1}$ for $\mathrm{HCO}^{+} J=3-2$ and $\mathrm{N}_{2} \mathrm{H}^{+} J=3-2$, respectively.

To calculate the line fluxes for our model we use the line transfer module of PRoDiMo (Woitke et al. 2011) and the molecular data from the Leiden Atomic and Molecular Database (Schöier et al. 2005; Botschwina et al. 1993; Flower 1999). For our reference model CI_XN we find fluxes of $2.4 \mathrm{Jy} \mathrm{km} \mathrm{s}^{-1}$ and $0.78 \mathrm{Jy} \mathrm{km} \mathrm{s}^{-1}$ for $\mathrm{HCO}^{+} J=3-2$ and $\mathrm{N}_{2} \mathrm{H}^{+} J=3-2$, respectively. As the sample of Öberg et al. (2011a) is probably biased towards large and massive disks we also calculated the fluxes for a disk four times more massive. We find fluxes of $4.3 \mathrm{Jy} \mathrm{km} \mathrm{s}^{-1}$ and $1.18 \mathrm{Jy} \mathrm{km} \mathrm{s}^{-1}$ for $\mathrm{HCO}^{+} J=3-2$ and $\mathrm{N}_{2} \mathrm{H}^{+} J=3-2$, respectively. These results are well within the range of the observations. 